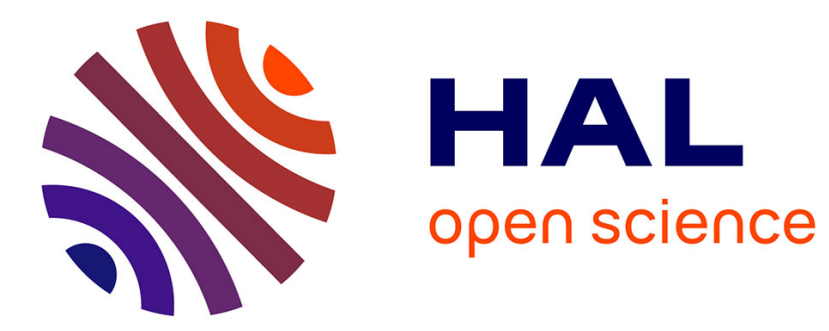

\title{
Application des lois de l'électromagnétisme, à l'étude des réseaux
}

R. Petit, D. Maystre

\section{To cite this version:}

R. Petit, D. Maystre. Application des lois de l'électromagnétisme, à l'étude des réseaux. Revue de Physique Appliquée, 1972, 7 (4), pp.427-441. 10.1051/rphysap:0197200704042700 . jpa-00243648

\section{HAL Id: jpa-00243648 https://hal.science/jpa-00243648}

Submitted on 1 Jan 1972

HAL is a multi-disciplinary open access archive for the deposit and dissemination of scientific research documents, whether they are published or not. The documents may come from teaching and research institutions in France or abroad, or from public or private research centers.
L'archive ouverte pluridisciplinaire HAL, est destinée au dépôt et à la diffusion de documents scientifiques de niveau recherche, publiés ou non, émanant des établissements d'enseignement et de recherche français ou étrangers, des laboratoires publics ou privés. 
Classification

Physics Abstracts

$02.40,05.10,08.20,08.40$

\title{
APPLICATION DES LOIS DE L'ÉLECTROMAGNÉTISME, A L'ÉTUDE DES RÉSEAUX
}

\author{
R. PETIT et D. MAYSTRE \\ Faculté des Sciences, St-Jérôme, 13, Marseille (13e) \\ (Reçu le 4 avril 1972, révisé le 26 juin 1972)
}

\begin{abstract}
Résumé. - Quand le pas est de l'ordre de grandeur de la longueur d'onde certaines propriétés des réseaux se révèlent très sensibles à la polarisation. Il faut alors tenir compte du caractère vectoriel de l'onde électromagnétique incidente. Cet article est un exposé de mise au point sur les travaux effectués dans ce domaine depuis une dizaine d'années.

Abstract. - If the period of a grating is of the same order as the wavelength, some of its properties depend on the polarization. Scalar theories cannot be used and we have to take into account the vector character of the incident electromagnetic wave. This paper reviews the differents works which have been done on the subject during the last ten years.
\end{abstract}

La théorie des réseaux de diffraction, traditionnellement enseignée en Optique, est devenue au cours des dix dernières années un important thème de recherche en Electromagnétisme. Les réseaux actuellement utilisés dans le visible ont en effet suffisamment de traits par millimètre pour que leur pas soit du même ordre de grandeur que la longueur d'onde. Dans ces conditions, certaines de leurs propriétés sont très sensibles à la polarisation et les théories scalaires de l'Optique doivent faire place aux théories vectorielles de l'Electromagnétisme. Il faut, à partir des équations de Maxwell, expliquer des phénomènes que l'on classe habituellement grâce à quatre mots-clés : diffraction, dispersion, résolution et luminosité. A vrai dire, ce sont surtout les questions de luminosité qui nécessitent le recours aux lois générales de l'électromagnétisme. Aussi, délaissant l'important problème de la résolution, nous supposerons toujours que le réseau, éclairé sous l'incidence $\theta$ par une onde plane, de longueur d'onde $\lambda=2 \pi / k$, est illimité et nous étudierons essentiellement la distribution de l'énergie entre les différentes ondes diffractées. Celle-ci dépend a priori de la polarisation et des paramètres géométriques et électromagnétiques caractérisant l'onde incidente et la surface diffractante. Nous décrirons successivement deux grands types de méthodes généralement appelées méthodes intégrales ou méthodes différentielles suivant que l'on ramène la détermination du champ diffracté à la résolution d'une équation intégrale ou d'un système d'équations différentielles couplées.

Dans tout l'article, l'espace est rapporté à un trièdre orthonormé $O x y z$ et $\mathbf{e}_{1}, \mathbf{e}_{2}, \mathbf{e}_{3}$ sont les vecteurs unitaires de $O x, O y, O z$. Les traits du réseau sont toujours parallèles à $O z$ et le plus souvent la surface diffractante est une surface cylindrique d'équation $y=f(x /$. Dans ce cas, le pas $d=2 \pi / K$ est la période de la fonction $f(x)$ dont le graphe $\mathfrak{T}$ (profil) décrit la forme des sillons; $\mathbf{n}$ est le vecteur unitaire de la normale à $\mathfrak{T}$ dirigé vers le demi-espace $y>f(x)$ supposé vide (Fig. 1). Toute grandeur scalaire sinusoïdale $a \cos (\omega t-\varphi)$ est systématiquement représentée par son amplitude $a \exp (\mathrm{i} \varphi)$ ce qui suppose une dépendance temporelle en $\exp (-i \omega t)$.

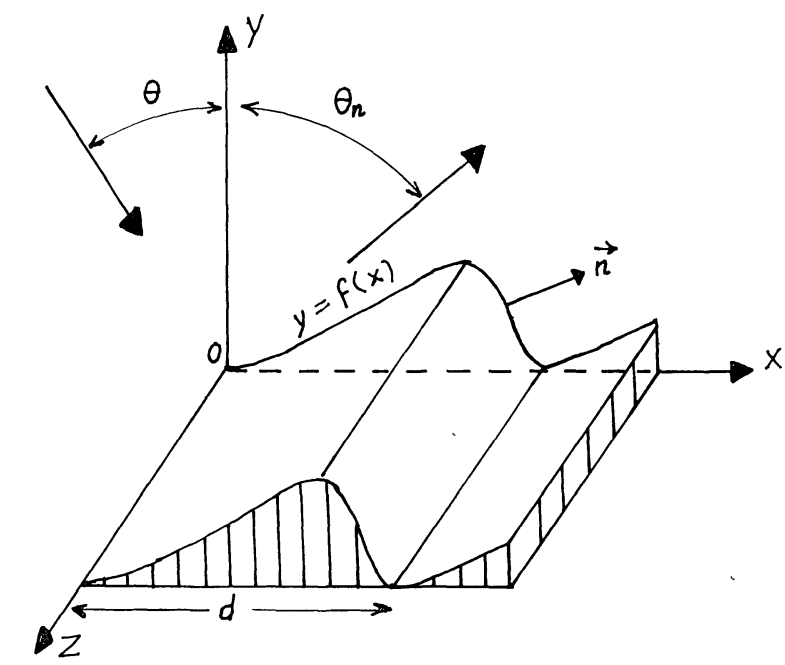

FIG. 1. - Notations utilisées : $\theta$ est l'angle d'incidence, $\theta_{n}$ l'angle de diffraction d'ordre $n$; avec nos conventions, $\theta$ et $\theta_{n}$ sont ici positifs.

I. Le formalisme intégral. - Bien que quelques publications traitent des réseaux diélectriques ou conducteurs [1], [2], nous nous limiterons à la théorie des réseaux infiniment conducteurs qui, sauf peutêtre en ultra-violet, représentent convenablement les réseaux utilisés en Optique. Plutôt que de décrire chronologiquement les travaux des différents auteurs 
qui ont contribué à l'élaboration de cette théorie, nous essayons de présenter un exposé synthétique en utilisant la théorie des distributions [3], [4] qui nous semble l'outil mathématique susceptible de concilier rigueur et concision $\left({ }^{1}\right)$. Les deux cas dits fondamentaux que nous étudions tout d'abord méritent cette appellation car, comme on le verra, leur résolution permet celle du cas général.

I.1 LE PREMIER CAS FONDAMENTAL : $\mathbf{E} \|$. I.1.1 Définition. - Le réseau est décrit sur la figure 1 : la région $y<f(x)$ est remplie d'un métal infiniment conducteur à l'intérieur duquel le champ électromagnétique est nul en régime permanent; le champ électrique $\mathbf{E}^{\mathbf{i}}$ de l'onde plane incidente est parallèle à $O z$ :

$\mathbf{E}^{\mathbf{i}}(x, y)=E^{\mathbf{i}}(x, y) \mathbf{e}_{3}=\exp (i k(x \sin \theta-y \cos \theta)) \mathbf{e}_{3}$.

Il donne naissance au-dessus du réseau à un champ électromagnétique $\mathbf{E}, \mathbf{H}$ de même pulsation.

I.1.2 Mise en équation et généralités (Fig. 2). -

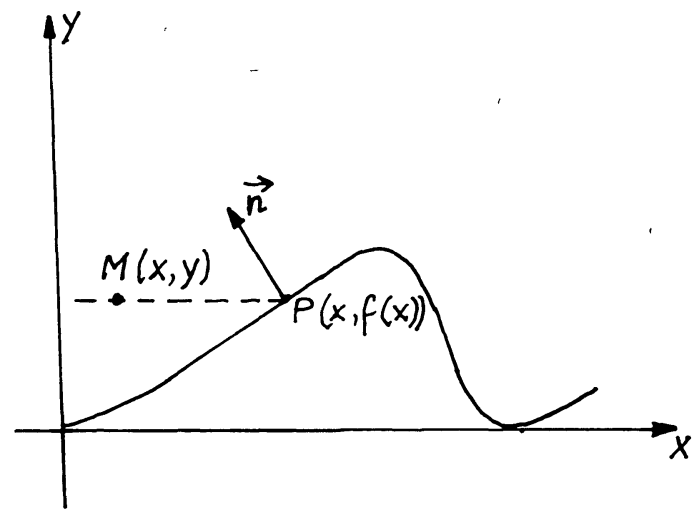

FIG. 2. - Etablissement des équations intégrales.

(1) Les distributions utilisées dans cet article sont des fonctionnelles linéaires sur un espace fonctionnel $\mathcal{R}_{\theta}$ adapté au problème du réseau éclairé sous une incidence $\theta$. Elles forment ellesmêmes un espace vectoriel noté $\boldsymbol{R}_{\boldsymbol{\theta}}^{\prime}$.

$\Re_{\theta}$ est l'ensemble des fonctions $\xi(x, y)$ indéfiniment dérivables à support borné en $y$ et telles que :

$$
\xi(x+d, y)=\xi(x, y) \exp (-i k d \sin \theta) .
$$

Désignant par $\mathfrak{D}^{\prime}(\Gamma)$ l'espace des distributions sur un cercle $\Gamma$ de longueur $d$ (Chap. IV de [3]), nous nous référons souvent à l'espace $\mathcal{R}_{0}$ 'des distributions sur un cylindre $\left(\mathcal{R}_{0}^{\prime}=\mathfrak{D}^{\prime}(\Gamma) \otimes \mathfrak{D}_{y}^{\prime}\right)$ et notons $\xi_{0}(x, y)$ les éléments de $\Re_{0}$.

Soit $\mathscr{F}$ l'application bijective qui associe à tout élément $T$ de $\mathcal{R}_{\theta}^{\prime}$ un élément $T_{0}$ de $\mathcal{R}_{0}^{\prime}$ :

$$
\begin{gathered}
T \stackrel{\mathscr{F}}{\longrightarrow} T_{0}, \quad T_{0} \stackrel{\mathscr{F}-1}{\longrightarrow} T ; \\
<T, \xi>=<T_{0}, \xi \exp (i k x \sin \theta)>, \\
<T_{0}, \xi_{0}>=<T, \xi_{0} \exp (-i k x \sin \theta)>.
\end{gathered}
$$

On vérifie sans peine que $\widetilde{F}$ définit entre $\mathcal{R}_{\theta}^{\prime}$ et $\mathcal{R}_{0}^{\prime}$ un isomorphisme qui respecte l'addition et la convolution.

On notera aussi que si $T_{0}$ est une fonction périodique $u_{0}(x, y)$, $T$ est la fonction "pseudo-périodique » $u(x, y)=u_{0}(x, y)$ $\exp (i k x \sin \theta)$. Ainsi

$$
u(x+d, y)=u(x, y) \exp (i k d \sin \theta)
$$

Il suffit de déterminer le champ électrique diffracté $\mathbf{E}^{\mathrm{d}}=\mathbf{E}-\mathbf{E}^{\mathrm{i}}$ qui vérifie :

- l'équation de Helmholtz

$$
\Delta \mathbf{E}^{\mathrm{d}}+k^{2} \mathbf{E}^{\mathrm{d}}=0 \quad \text { si } \quad y>f(x),
$$

- une condition d'ondes sortantes pour $y \rightarrow+\infty$,

- une condition aux limites résultant de la continuité de la composante tangentielle de $\mathbf{E}$ à la traversée de $\mathfrak{T}$ :

$$
\lim _{M \rightarrow P} \mathbf{n}(P) \Lambda \mathbf{E}^{\mathrm{d}}(M)=-\mathbf{n}(P) \Lambda \mathbf{E}^{\mathrm{i}}(P) .
$$

En admettant qu'en raison de son origine physique ce problème a une solution unique, on peut montrer que $\mathbf{E}^{\mathrm{d}}$ est nécessairement de la forme $E^{\mathrm{d}}(x, y) \mathbf{e}_{3}$, ce qui d'ailleurs est très intuitif.

Il s'agit donc de trouver la fonction scalaire $E^{\mathrm{d}}(x, y)$ vérifiant :

- l'équation scalaire de Helmholtz

$$
\Delta E^{\mathrm{d}}+k^{2} E^{\mathrm{d}}=0 \quad \text { si } \quad y>f(x),
$$

- une condition d'ondes sortantes pour $y \rightarrow+\infty$

- une condition aux limites :

$$
\lim _{M \rightarrow P} E^{\mathrm{d}}(M)=-E^{\mathrm{i}}(P) .
$$

Soit $u(x, y)$ la fonction égale à $E^{\mathrm{d}}$ si $y>f(x)$ et à $-E^{i}$ si $y<f(x)$. C'est une fonction continue qui satisfait une condition d'ondes sortantes pour $y \rightarrow \pm \infty$ et vérifie au sens des distributions $\left({ }^{2}\right)$ :

$$
\Delta u+k^{2} u=\varphi \delta_{\mathfrak{T}}
$$

où la fonction $\varphi$ définie sur $\mathfrak{T}$ est le saut de la dérivée normale de $u$ en franchissant $\mathcal{T}$ dans le sens de $\mathbf{n}$. Les équations de Maxwell et les conditions de passage associées permettent d'ailleurs de relier $\varphi$ au courant superficiel $j_{\mathrm{S}} \delta_{\mathfrak{T}} \mathbf{e}_{3}$. On trouve que $\varphi=-i \omega \mu_{0} j_{\mathrm{S}}$ et ceci est le point de départ de la formulation proposée par J. Pavageau [5].

Ainsi, en prolongeant naturellement le champ diffracté par l'opposé du champ incident (ce qui revient à prolonger le champ total par zéro), nous nous ramenons à la détermination d'une fonction $u(x, y)$ vérifiant :

$$
\Delta u+k^{2} u=\varphi \delta_{\mathfrak{T}},
$$

une condition d'ondes sortantes pour

$$
\begin{aligned}
y & \rightarrow \pm \infty, \\
u(x, f(x)) & =-E^{\mathrm{i}}(x, f(x)) .
\end{aligned}
$$

(2) $\delta_{\mathfrak{T}}$ est un symbole désignant l'élément de $\Re_{\theta}^{\prime}$ associé à l'élément $\varphi \exp (-i k x \sin \theta) \delta_{\mathfrak{T}}$ de $\mathcal{R}_{0}^{\prime}: \operatorname{si} \xi_{0} \in \mathcal{R}_{0}$,

$$
\begin{aligned}
&<\varphi \exp (-i k x \sin \theta) \delta \mathfrak{T}, \xi_{0}>= \\
&=\int \varphi \exp (-i k x \sin \theta) \xi_{0}(x, f(x)) \mathrm{d} s,
\end{aligned}
$$

où $s$ désigne l'abscisse curviligne sur une période $\mathfrak{T}$. 
On verra, en se reportant à [6], qu'une telle fonction est unique et que, comme $E^{\mathbf{i}}$, elle est « pseudo-périodique ». Elle est donc un élément de l'espace $\mathcal{R}^{\prime}$ défini dans la première note de bas de page.

Dès lors, si nous sommes capables de trouver la fonction $G(x, y)$ appartenant à $\mathcal{R}^{\prime}$, vérifiant (2) et solution de $\left({ }^{3}\right)$ :

$$
\Delta G+k^{2} G=\delta
$$

on pourra exprimer $u$ en fonction de $\varphi$ par le produit de convolution $G * \varphi \delta_{\mathfrak{T}}$ qui vérifie (2) et (1) puisque :

$$
\begin{aligned}
\left(\Delta+k^{2}\right) G * \varphi \delta_{\mathfrak{T}} & =\left(\Delta G+k^{2} G\right) * \varphi \delta_{\mathfrak{T}}, \\
& =\delta * \varphi \delta_{\mathfrak{T}}=\varphi \delta_{\mathfrak{T}} .
\end{aligned}
$$

Autrement dit, $G$ et $\varphi$ étant supposées connues, la fonction $u$, qui s'identifie à $E^{\mathrm{d}}$ au-dessus du réseau, est donnée par :

$$
u=G * \varphi \delta_{\mathfrak{T}}=\left(G_{0} * \varphi_{0} \delta_{\mathfrak{T}}\right) \exp (i k x \sin \theta),
$$

$$
\begin{aligned}
& u(x, y)=\exp (i k x \sin \theta) \int \varphi_{0}\left(x^{\prime}\right) \times \\
& \times G_{0}\left(x-x^{\prime}, y-f\left(x^{\prime}\right)\right) \mathrm{d} s^{\prime},
\end{aligned}
$$

l'intégration étant effectuée sur une période par rapport à l'abscisse curviligne.

I.1.3 Détermination de $G_{0}$. - Si $G_{0}(x, y)$ est la fonction de $\mathcal{R}_{0}^{\prime}$ associée à $G(x, y)$, on sait que puisque $G_{0}$ est périodique en $x$ :

$$
G_{0}(x, y)=\sum_{n=-\infty}^{+\infty} g_{n}(y) \exp (i n K x)
$$

On en déduit que :

$$
\begin{aligned}
G(x, y)=\sum_{n=-\infty}^{+\infty} g_{n}(y) \exp (i x(n K+ & k \sin \theta))= \\
& =\sum_{n=-\infty}^{+\infty} g_{n}(y) e_{n}(x)
\end{aligned}
$$

avec $e_{n}(x)=\exp (i x(n K+k \sin \theta))$

De la même façon, puisque

$$
\delta_{0}=\delta(y) \sum_{n=-\infty}^{+\infty} \frac{1}{d} \exp (i n K x)
$$

nous pouvons écrire :

$$
\begin{aligned}
\delta=\sum_{n=-\infty}^{+\infty} \frac{\delta(y)}{d} \exp (i x(n K+k \sin \theta)) & \\
& =\sum_{n=-\infty}^{+\infty} \frac{\delta(y)}{d} e_{n}(x) .
\end{aligned}
$$

En reportant ces deux développements dans les deux

(3) $\delta$ que l'on peut appeler distribution de Dirac dans $\Re^{\prime}$ est associée à la distribution de Dirac $\delta_{0}$ sur le cylindre :

$$
\delta_{0}=\delta(y) \sum_{n=-\infty}^{+\infty} \frac{1}{d} \exp (\text { inK } K)
$$

membres de (4) et en identifiant les coefficients de $e_{n}(x)$, on est conduit à :

$$
\frac{d^{2} g_{n}}{d y^{2}}+k^{2}\left(1-\alpha_{n}^{2}\right) g_{n}=\frac{1}{d} \delta(y)
$$

où $\alpha_{n}=\sin \theta+n(K / k)$.

Ceci montre que $g_{n}(y)$ est une fonction continue dont la dérivée présente un saut égal à $1 / d$ pour $y=0$. En se souvenant que $G(x, y)$ doit satisfaire une condition d'ondes sortantes pour $y \rightarrow \infty$ et en posant $\beta_{n}^{2}=1-\alpha_{n}^{2}$, on trouve facilement [6]:

$$
g_{n}(y)=\frac{1}{2 i d k \beta_{n}} \exp \left(i k \beta_{n}|y|\right),
$$

expression dans laquelle il convient de noter la présence du symbole valeur absolue. Nous venons d'obtenir un résultat fondamental pour tous les problèmes de diffraction par un réseau :

$$
G_{0}(x, y)=\frac{1}{2} \frac{1}{i d k} \sum_{n=-\infty}^{+\infty} \frac{1}{\beta_{n}} \exp \left(i n K x+i k \beta_{n}|y|\right) \text {. }
$$

I.1.4 Détermination de $\varphi$. $-G_{0}$ étant connue, $\varphi_{0}$ peut-être déterminée grâce à l'équation (3) qui impose :

$$
\begin{aligned}
\int \varphi_{0}\left(x^{\prime}\right) G_{0} & \left(x-x^{\prime}, f(x)-f\left(x^{\prime}\right)\right) \mathrm{d} s^{\prime}= \\
= & \int \varphi_{0}\left(x^{\prime}\right) N_{1}\left(x, x^{\prime}\right) \mathrm{d} x^{\prime}=-E_{0}^{i}(x, f(x)) \\
& =-\exp (-i k f(x) \cos \theta)
\end{aligned}
$$

Il s'agit d'une équation intégrale de Fredholm et de première espèce dont le noyau $N_{1}\left(x, x^{\prime}\right)$ est singulier mais intégrable [6], [7].

Une autre façon de procéder utilisée par J. Pavageau [5] consiste à écrire (Fig. 2) la relation liant $j_{\mathrm{S}}$ au champ magnétique :

$$
\lim _{M \rightarrow P}(\mathbf{n}(P) \Lambda \mathbf{H}(M))=j_{S}(P) \mathbf{e}_{3} .
$$

En utilisant les équations de Maxwell et en posant $u_{\mathrm{t}}=u+E^{\mathrm{i}}$, on arrive à la relation scalaire :

$$
\lim _{M \rightarrow P}\left[\mathbf{n}(P) \cdot \operatorname{grad} u_{\mathrm{t}}(M)\right]=-i \omega \mu_{0} j_{\mathrm{S}}(P) .
$$

En explicitant $u_{\mathrm{t}}$ compte tenu de (5) et (8) et en permutant les signes $\int$ et $\sum$, il apparaît que l'expression figurant entre crochets au premier membre de (10) est, pour $P$ fixé, un développement sur la base $e_{n}(x)$. Mais il s'agit du développement d'une fonction a priori discontinue de sorte que la valeur prise par ce développement au point $P$ est égale à la demi-somme de sa valeur à droite et de sa valeur à gauche $\left({ }^{4}\right)$. Sa valeur

(4) Ceci se déduit immédiatement d'un théorème sur les séries de Fourier; la série de Fourier d'une fonction converge vers la moyenne arithmétique de sa valeur limite à gauche et de sa valeur limite à droite. 
dans le métal (à droite de $P$ sur la figure 2) étant nulle puisque dans le métal $u_{\mathrm{t}}=0$, on peut écrire :

$\frac{1}{2}\left[0+\lim _{M \rightarrow P}\left(\mathbf{n}(P) \Lambda \operatorname{grad} u_{\mathrm{t}}(M)\right)\right]=\mathbf{n}(P) \cdot \operatorname{grad} u_{\mathrm{t}}(P)$,

sous réserve d'existence de l'intégrale qui apparaît au second membre compte tenu de (5). En comparant (11) et (10), il vient :

$$
-\frac{i}{2} \omega \mu_{0} j_{\mathrm{S}}(P)=\mathbf{n}(P) \cdot \operatorname{grad} u_{t}(P) .
$$

Il suffit de remplacer $u_{\mathrm{t}}$ par $u+E^{\mathrm{i}}$ et d'expliciter $u$ grâce à (5) et (8) pour obtenir une équation intégrale pour $\varphi$.

On allège l'écriture en posant :

$$
\begin{aligned}
\hat{\varphi}(x) & =\varphi(x) \sqrt{1+f^{\prime 2}(x)}=\widehat{\varphi}_{0}(x) \exp (+i k x \sin \theta), \\
\eta(x) & =-2 i k\left(\cos \theta+f^{\prime}(x) \sin \theta\right) E^{\mathrm{i}}(x, f(x)) \\
& =\eta_{0}(x) \exp (+i k x \sin \theta) .
\end{aligned}
$$

On arrive ainsi à une équation de Fredholm de deuxième espèce :

$$
\widehat{\varphi}_{0}(x)=n_{0}(x)+\int N_{2}\left(x, x^{\prime}\right) \widehat{\varphi}_{0}\left(x^{\prime}\right) \mathrm{d} x^{\prime},
$$

avec :

$$
\begin{aligned}
& N_{2}\left(x, x^{\prime}\right)=\frac{1}{d} \sum_{n=-\infty}^{+\infty}\left(\operatorname{sgn}\left(f(x)-f\left(x^{\prime}\right)\right)-\right. \\
& \left.-\frac{\alpha_{n}}{\beta_{n}} f^{\prime}(x)\right) \exp \left(i n K\left(x-x^{\prime}\right)+i k \beta_{n}\left|f(x)-f\left(x^{\prime}\right)\right|\right)
\end{aligned}
$$

noyau dont les singularités et l'intégrabilité ont été étudiées par J. Pavageau [5].

I. 2 LE DEUXiÈme CAS FONDAMENTAL : H H. I.1.2 Définition. - Le champ magnétique $\mathbf{H}$ est parallèle à $O z$; il suffit de reprendre le texte du paragraphe I.1.1 en remplaçant $\mathbf{E}^{\mathbf{i}}$ par $\mathbf{H}^{\mathbf{i}}$.

I.2.2 Mise en équation et généralités. - On raisonne sur $\mathbf{H}=\mathrm{He}_{3}$ et par analogie avec le cas précédent on s'intéresse à la fonction $u(x, y)$ égale à $H$ pour $y>f(x)$ et à $-H^{\mathrm{i}}$ pour $y<f(x)$.

Sa dérivée normale est contenue puisque dans le vide, au voisinage du réseau $\mathrm{d} H^{\mathrm{d}} / \mathrm{d} n=-\mathrm{d} H^{\mathrm{i}} / \mathrm{d} n$. On a donc, au sens des distributions :

$$
\Delta u+k^{2} u=\operatorname{div}\left(\psi \mathbf{n} \delta_{\mathfrak{T}}\right) .
$$

La fonction $\psi$ qui représente le saut de $u$ ou du champ total $u_{\mathrm{t}}=u+H^{\mathrm{i}}$ s'identifie d'ailleurs au courant superficiel $j_{\mathrm{s}}$ porté dans ce cas par la tangente à $\mathcal{T}$ $\left(\mathbf{j}_{\mathrm{S}}=j_{\mathrm{s}} \mathbf{t}\right)$.

En résumé, $u$ vérifie :

$$
\Delta u+k^{2} u=\operatorname{div}\left(\psi \mathbf{n} \delta_{\mathfrak{T}}\right),
$$

une condition d'ondes sortantes pour

$$
\begin{aligned}
y & \rightarrow \pm \infty, \\
\lim _{M \rightarrow P} u_{\mathrm{t}}(M) & =\psi(P)=j_{\mathrm{S}}(P) .
\end{aligned}
$$

La relation $\left(1^{\prime}\right)$ permet encore de représenter $u$ par un produit de convolution :

$$
\begin{array}{rl}
u(x, y)=G & * \operatorname{div}\left(\psi \mathbf{n} \delta_{\mathfrak{T}}\right)= \\
& =G_{0} *\left(\operatorname{div}\left(\psi \mathbf{n} \delta_{\mathfrak{T}}\right)\right)_{0} \exp (i k x \sin \theta),
\end{array}
$$

où $\left(\operatorname{div}\left(\psi \mathbf{n} \delta_{\mathfrak{T}}\right)\right)_{0}$ est la distribution de $\mathcal{R}_{0}^{\prime}$ associée à $\operatorname{div}\left(\psi \mathbf{n} \delta_{\mathfrak{T}}\right)$, c'est-à-dire $\left(^{5}\right)$ :

$$
\operatorname{div}\left(\mathbf{n} \psi_{0} \delta_{\mathfrak{J}}\right)+i k \sin \theta n_{x} \psi_{0} \delta_{\mathfrak{T}} .
$$

En explicitant, on obtient $u$ (et par suite $u_{\mathrm{t}}=u+H^{\mathrm{i}}$ ) sous forme d'un développement sur la base

$$
e_{n}(x)=\exp \left(i \alpha_{n} x\right)
$$

et le raisonnement employé au paragraphe I.1.4 montre que :

$$
\frac{1}{2}\left[0+\lim _{M \rightarrow P} u_{\mathrm{t}}(M)\right]=u_{\mathrm{t}}(P) .
$$

En tenant compte de $\left(3^{\prime}\right)$, on en déduit :

$$
\frac{1}{2} \psi(P)=u(P)+H^{\mathrm{i}}(P) .
$$

Tous calculs effectués, on obtient l'équation intégrale de deuxième espèce :

$$
\psi_{0}(x)=2 H_{0}^{\mathrm{i}}(x)+\int N_{3}\left(x, x^{\prime}\right) \psi_{0}\left(x^{\prime}\right) \mathrm{d} x^{\prime}
$$

(5) En appliquant les définitions données dans la première note de bas de page :

$$
\begin{aligned}
\left\langle\left(\frac{\partial}{\partial x}\left(\psi n_{x} \delta \mathfrak{T}\right)\right)_{0}, \xi_{0}\right\rangle= & \\
& =\left\langle\frac{\partial}{\partial x}\left(\psi n_{x} \delta \mathfrak{T}\right), \xi_{0} \exp (-i k x \sin \theta)\right\rangle, \\
& =-\left\langle\psi n_{x} \delta \mathfrak{T}, \frac{\partial}{\partial x}\left(\xi_{0} \exp (-i k x \sin \theta)\right)\right\rangle, \\
& =-\left\langle\psi_{0} n_{x} \delta \mathfrak{T}, \exp (i k x \sin \theta) \frac{\partial}{\partial x}(-i k x \sin \theta)\right\rangle, \\
& =-\left\langle\psi_{0} n_{x} \delta \mathfrak{T}, \frac{\partial \xi_{0}}{\delta x}-i k \sin \theta \xi_{0}\right\rangle, \\
& =\left\langle\frac{\partial}{\partial x}\left(\psi_{0} n_{x} \delta \mathfrak{T}\right)+i k \sin \theta \psi_{0} n_{x} \delta_{\mathfrak{T}}, \xi_{0}\right\rangle .
\end{aligned}
$$

Ainsi :

$$
\left(\frac{\partial}{\partial x}\left(\psi n_{x} \delta \mathfrak{T}\right)\right)_{0}=\frac{\partial}{\partial x}\left(\psi_{0} n_{x} \delta_{\mathfrak{T}}\right)+i k \sin \theta \psi_{0} n_{x} \delta \mathfrak{T} .
$$

On montre de la même façon que :

$$
\left(\frac{\partial}{\partial y}\left(\psi n_{y} \delta \mathfrak{T}\right)\right)_{0}=\frac{\partial}{\partial y}\left(\psi_{0} n_{y} \delta_{\mathfrak{T}}\right)
$$

d'où :

$(\operatorname{div}(\psi \mathbf{n} \delta \mathfrak{T}))_{0}=$

$$
\begin{aligned}
& =\frac{\partial}{\partial y}\left(\psi_{0} n_{x} \delta_{\mathfrak{T}}\right)+i k \sin \theta \psi_{0} n_{x} \delta_{\mathfrak{T}}+\frac{\partial}{\partial y}\left(\psi_{0} n_{y} \delta_{\mathfrak{T}}\right) . \\
& =\operatorname{div}\left(\psi_{0} \mathbf{n} \delta \mathfrak{T}\right)+i k \sin \theta \psi_{0} n_{x} \delta \mathfrak{T} .
\end{aligned}
$$


avec :

$$
\begin{aligned}
N_{3}\left(x, x^{\prime}\right) & =\frac{1}{d} \sum_{n=-\infty}^{+\infty}\left[\operatorname{sgn}\left(f(x)-f\left(x^{\prime}\right)\right)-\frac{\alpha_{n}}{\beta_{n}} f^{\prime}\left(x^{\prime}\right)\right] \times \\
& \times \exp \left(i n K\left(x-x^{\prime}\right)+i k \beta_{n}\left|f(x)-f\left(x^{\prime}\right)\right|\right) \quad\left(13^{\prime}\right)
\end{aligned}
$$

noyau dont les singularités et l'intégrabilité ont été étudiées par J. Pavageau [5].

I. 3 FORME DU CHAMP DIFFRACTÉ. - Le champ diffracté donné pour $y>f(x)$ par $(5)$ ou $\left(5^{\prime}\right)$ prend une forme simple si $y>\max f(x)$, région qui seule intéresse l'Opticien. Dans cette région, en effet, le symbole valeur absolue qui figure dans

$$
G\left(x-x^{\prime}, y-f\left(x^{\prime}\right)\right)
$$

peut être supprimé puisque

$$
\left|f(x)-f\left(x^{\prime}\right)\right|=f(x)-f\left(x^{\prime}\right) .
$$

Tout calcul fait, (5) et $\left(5^{\prime}\right)$ deviennent :

$$
\begin{aligned}
& E^{\mathrm{d}}=\sum_{n=-\infty}^{+\infty} B_{n}^{1} \exp \left(i k\left(\alpha_{n} x+\beta_{n} y\right)\right), \\
& H^{\mathrm{d}}=\sum_{n=-\infty}^{+\infty} B_{n}^{2} \exp \left(i k\left(\alpha_{n} x+\beta_{n} y\right)\right),
\end{aligned}
$$

où les coefficients $B_{n}^{1}$ et $B_{n}^{2}$ sont des complexes s'exprimant simplement en fonction de $\hat{\varphi}_{0}$ ou $\psi_{0}$ :

$$
\begin{aligned}
B_{n}^{1}= & \frac{1}{2 i d k \beta_{n}} \int \exp \left(-i n K x^{\prime}+i k \beta_{n} f\left(x^{\prime}\right)\right) \hat{\varphi}_{0}\left(x^{\prime}\right) \mathrm{d} x^{\prime} \\
B_{n}^{2}= & \frac{1}{2 d} \int\left[1-\frac{\alpha_{n}}{\beta_{n}} f^{\prime}\left(x^{\prime}\right)\right] \times \\
& \quad \times \exp \left(-i n K x^{\prime}+i k \beta_{n} f\left(x^{\prime}\right)\right) \psi_{0}\left(x^{\prime}\right) \mathrm{d} x^{\prime} .
\end{aligned}
$$

On retrouve donc le développement en ondes planes utilisé depuis Lord Rayleigh [8] :

Si $\alpha_{n}=\sin \theta+n(\lambda / d)$ est en module inférieur à 1 , on peut poser :

$$
\sin \theta_{n}=\sin \theta^{1}+n \frac{\lambda}{d}, \quad-\frac{\pi}{2}<\theta_{n}<\frac{\pi}{2},
$$

alors

$$
\beta_{n}=\sqrt{1-\alpha_{n}^{2}}=\cos \theta_{n}
$$

et

$$
B_{n} \exp \left(i k\left(x \sin \theta_{n}+y \cos \theta_{n}\right)\right)
$$

représente une onde plane diffractée sous l'angle $\theta_{n}$ (Fig. 1) que l'on calcule par la classique formule des réseaux (16).

$\mathrm{Si}$, au contraire, $\sin \theta+n(\lambda / d)$ est en module supérieur à $1, \beta_{n}$ est imaginaire pur et

$$
B_{n} \exp \left(i k \alpha_{n} x+i k \beta_{n} y\right)
$$

représente une «onde évanescente », exponentiellement amortie suivant $O y$, qui se propage perpendiculairement aux sillons.

On a longtemps cru que le développement. de Rayleigh était susceptible de représenter partout (même pour $f(x)<y<\max f(x))$ le champ diffracté et c'est à partir de cette hypothèse, grâce à la vulgarisation du calcul sur ordinateur, que les premiers calculs d'efficacité (détermination des $B_{n}$ ) furent effectués ([9] à [17]) en dépit d'une mise en garde de Lippmann [18]. La question de la validité du développement de Rayleigh a donné lieu à des discussions passionnées au cours des dix dernières années et surtout après la publication en 1966 d'une note de Petit et Cadilhac. Il semble qu'aujourd'hui, après les travaux de Millar [20], [21], Nevière et Cadilhac [22], Barentsev [23] et Wirgin [24], la cause défendue par Lippmann soit entendue : le développement en ondes planes n'est utilisable que dans des conditions très particulières liées au profil du réseau ; il ne peut donc pas servir de base à un travail qui aurait l'ambition d'être général. Par exemple, quelques cas très singuliers mis à part, il n'est pas valable pour un réseau échelette [22] tandis que pour un réseau sinusoïdal $(y=h \cos (2 \pi x / d))$ sa validité suppose $h<0,072 d[21]$. Il faut cependant signaler que son utilisation dans un domaine théoriquement interdit permet parfois d'obtenir des résultats numériques approchés mais satisfaisants [6], [19].

Ainsi, loin du réseau, dans la région intéressante pour l'Optique, le champ diffracté a une forme simple qui peut être caractérisée par la donnée d'une suite de coefficients $B_{n}$. La détermination de ces coefficients ne peut malheureusement se faire qu'en écrivant une condition aux limites sur le réseau c'est-à-dire dans une région où cette forme simple ne peut pas en général représenter le champ. C'est là le drame du problème $d u$ réseau et c'est avec peine que beaucoup d'Opticiens ont dû renoncer au développement de Rayleigh définitivement condamné par l'ordinateur: ce sont en effet les échecs enregistrés lors des premiers calculs [14] qui ont fait prendre en considération ([6], [24]) la remarque déjà ancienne de Lippmann.

I. 4 RÉSOLUTION NUMÉRIQUE DES ÉQUATIONS INTÉGRALES. - Le formalisme proposé dans les paragraphes $I .1$ et $I .2$ ramène la détermination du champ diffracté à celle d'une fonction $J$ liée au courant superficiel. Cette fonction qui s'identifie à $\hat{\varphi}_{0}$ ou $\psi_{0}$ suivant la polarisation est solution d'une équation intégrale de Fredholm. Cette équation peut être de première espèce :

$$
\int N\left(x, x^{\prime}\right) J\left(x^{\prime}\right) \mathrm{d} x^{\prime}=S(x),
$$

ou de seconde espèce :

$$
J(x)+\int N\left(x, x^{\prime}\right) J\left(x^{\prime}\right)=S(x),
$$

mais dans les deux cas le noyau $N\left(x, x^{\prime}\right)$, le second membre connu $S(x)$ et l'inconnue $J$ sont des fonctions périodiques de la variable $x$. Deux types de méthodes semblent avoir été utilisées.

I.4.1. - On représente $J(x)$ par les coefficients $J_{n}$ de son développement sur la base $\exp ($ inKx). 
Par projection de (17) ou $\left(17^{\prime}\right)$ sur cette base, on obtient un système linéaire infini qui, pour l'éq. (17), s'écrit :

$$
\sum_{m=-\infty}^{+\infty} a_{n m} J_{m}=S_{n}
$$

avec

$$
a_{n m}=\iint N\left(x, x^{\prime}\right) \exp \left(i m K x^{\prime}-i n K x\right) \mathrm{d} x \mathrm{~d} x^{\prime},
$$

et

$$
S_{n}=\int S(x) \exp (-i n K x) \mathrm{d} x
$$

Ce système devient un système de rang $N$ si l'on admet que $J(x)$ est convenablement représentée par $N$ termes de son développement. C'est la «méthode des réduites » [14] dont la validité suppose évidemment la convergence des approximations successivement obtenues en donnant à $N$ des valeurs de plus en plus grandes. Il faut noter que le calcul des $a_{n m}$ est celui de la somme d'une série d'intégrales doubles. La méthode est donc surtout intéressante quand ces dernières se calculent analytiquement ; cela se produit pour les réseaux échelettes et plus généralement chaque fois que le profil est décrit par un petit nombre de segments de droite [6].

I.4.2. - On représente $J(x)$ par ses valeurs en $N$ points $x_{n}(n=1, \ldots, N)$ de l'intervalle $(0, d)$. Cette discrétisation conduit à un système linéaire de rang $N$ pour les inconnues $J\left(x_{n}\right)$. La résolution sur ordinateur est effectuée, suivant les auteurs, par un processus itératif dont la convergence n'est pas théoriquement assurée [5], [25] ou par utilisation de programmes classiques (méthode du pivot maximum, par exemple). La tabulation du noyau nécessite la sommation laborieuse de séries dont plusieurs auteurs ont essayé d'améliorer la vitesse de convergence [1], [2], [26].

I. 5 CRITÈres DE VALIDITÉ DES RÉSUltạTS NUMÉRIQUES OBTENUS. - Les calculs précédemment décrits sont, à vrai dire, assez volumineux. Il importe donc de pouvoir tester rapidement la validité des résultats numériques fournis par l'ordinateur. On peut par exemple vérifier que le champ calculé s'identifie à l'opposé du champ incident pour $y<f(x)$. Un critère plus rapide est fourni par la conservation de l'énergie qui impose :

$$
\sum_{n} B_{n} \bar{B}_{n} \frac{\cos \theta_{n}}{\cos \theta}=1
$$

cette somme étant étendue aux seules ondes planes et non aux ondes évanescentes [6], [27]. L'expérience numérique apprend qu'il est relativement facile de vérifier (19) avec une précision de l'ordre du centième et ceci nécessite des temps de calcul de l'ordre de quelques secondes sur UNIVAC 1108.

I. 6 CAS GÉNÉRAL. - Nous avons jusqu'ici supposé le vecteur d'onde incidente orthogonal aux sillons, mais le cas général a été récemment résolu dans notre laboratoire [28], [29] et par P. M. van der Berg [1]. La méthode utilisée par van der Berg nous semblant la plus élégante, nous allons nous en inspirer $\left({ }^{6}\right)$ pour montrer que le cas le plus général se ramène effectivement aux deux cas fondamentaux notés $\mathbf{E} / /$ et $\mathbf{H} / /$ dans les paragraphes précédents.

Soient $\boldsymbol{E}^{\mathbf{i}}$ et $\mathfrak{J}^{\mathbf{i}}$ les champs associés à l'onde plane incidente se propageant suivant la direction $\mathbf{u}$ de paramètres directeurs $\alpha,-\beta, \gamma(\alpha, \beta, \gamma$ positifs, $\left.\alpha^{2}+\beta^{2}+\gamma^{2}=1\right):$

$$
\begin{gathered}
\boldsymbol{\delta}^{\mathbf{i}}=\mathbf{a} \exp (i k(\alpha x-\beta y+\gamma z)), \\
\alpha a_{x}-\beta a_{y}+\gamma a_{z}=0, \quad a_{x}^{2}+a_{y}^{2}+a_{z}^{2}=1, \\
\mathfrak{J}^{\mathbf{i}}=\sqrt{\frac{\varepsilon_{0}}{\mu_{0}}} \frac{\mathbf{k}}{k} \Lambda \boldsymbol{\delta}^{\mathrm{i}} .
\end{gathered}
$$

En admettant l'existence et l'unicité de la solution, on montre [29] que, comme le champ incident, le champ diffracté $\left(\boldsymbol{E}^{\mathrm{d}}, \mathfrak{H}^{\mathrm{d}}\right)$ et le champ total $(\boldsymbol{E}, \boldsymbol{H})$ sont le produit d'une fonction des deux variables $x$ et $y$ par $\exp (i k \gamma z)$ :

$$
\begin{aligned}
\boldsymbol{E}^{\mathrm{i}}(x, y, z) & =\mathbf{E}^{\mathrm{i}}(x, y) \exp (i k \gamma z), \\
\boldsymbol{E}^{\mathrm{d}}(x, y, z) & =\mathbf{E}^{\mathrm{d}}(x, y) \exp (i k \gamma z), \\
\mathscr{H}^{\mathrm{i}}(x, y, z) & =\mathbf{H}^{\mathrm{i}}(x, y) \exp (i k \gamma z), \\
\mathscr{H}^{\mathrm{d}}(x, y, z) & =\mathbf{H}^{\mathrm{d}}(x, y) \exp (i k \gamma z) .
\end{aligned}
$$

Il suffit d'écrire que $\boldsymbol{\xi}^{\mathrm{d}}$ et $\boldsymbol{H}^{\mathrm{d}}$ vérifient l'équation de Helmholtz au-dessus du réseau et de tenir compte des conditions aux limites imposées par le métal infiniment conducteur pour trouver, en tenant compte des équations de Maxwell, que les fonctions $E_{z}^{\text {d }}$ et $H_{z}^{\mathrm{d}}$ sont chacune solution d'un cas fondamental.

On trouve par exemple que $E_{z}^{\mathrm{d}}$ qui vérifie une condition d'ondes sortantes pour $y \rightarrow+\infty$ vérifie aussi :

$$
\Delta E_{z}^{\mathrm{d}}+k^{2}\left(1-\gamma^{2}\right) E_{z}^{\mathrm{d}}=0, \text { pour } y>f(x)
$$

et

$$
E_{z}^{\mathrm{d}}(x, f(x))=-E_{z}^{\mathrm{i}}(x, f(x)) .
$$

Il est commode d'introduire les paramètres directeurs $\tilde{\alpha}$ et $-\tilde{\beta}$ de la projection de u sur le plan $x O y$ et le module $\tilde{k}$ de la projection de $\mathbf{k}$ sur ce même plan :

$$
\begin{aligned}
& \tilde{\alpha}=\frac{\alpha}{\sqrt{\alpha^{2}+\beta^{2}}}=\frac{\alpha}{\sqrt{1-\gamma^{2}}}, \\
& \tilde{\beta}=\frac{\beta}{\sqrt{\alpha^{2}+\beta^{2}}}=\frac{\beta}{\sqrt{1-\gamma^{2}}}, \\
& \tilde{\alpha}^{2}+\tilde{\beta}^{2}=1, \\
& \tilde{k}=k \sqrt{\left(\alpha^{2}+\beta^{2}\right)}=k \sqrt{1-\gamma^{2}}, \\
& \tilde{\lambda}=\frac{2 \pi}{\tilde{k}}=\frac{\lambda}{\sqrt{1-\gamma^{2}}} .
\end{aligned}
$$

(6) Bien qu'elle ne traite pas de la diffraction par les réseaux, nous avons aussi consulté avec profit la thèse de J. C. Bolomey [30]. 
D'après (24), ceci permet de considérer $E_{z}^{\mathrm{d}}$ comme le champ diffracté associé au champ incident

$$
E_{z}^{\mathrm{i}}=a_{z} \exp (i k(\alpha x-\beta y))=a_{z} \exp (i \tilde{k}(\tilde{\alpha} x-\tilde{\beta} y))
$$

pour un problème du type $\mathbf{E} / /$ dans lequel $k$ est remplacé par $\tilde{k}$. Il en résulte que pour $y>\max f(x)$ :

$$
E_{z}^{\mathrm{d}}=\sum_{n=-\infty}^{+\infty} a_{z} B_{n}^{1} \exp \left(i \tilde{k}\left(\tilde{\alpha}_{n} x+\tilde{\beta}_{n} y\right)\right)
$$

avec

$$
\tilde{\alpha}_{n}=\tilde{\alpha}+n \frac{\tilde{\lambda}}{d} \text { et } \quad \tilde{\beta}_{n}=\sqrt{1-\tilde{\alpha}_{n}^{2}} \text {. }
$$

Les coefficients $B_{n}^{1}$ s'obtiennent grâce au programme mis au point pour le cas $\mathbf{E} / /$ quand on remplace $k$ $\operatorname{par} \tilde{k}$ et $\alpha$ par $\tilde{\alpha}$.

De la même façon, $H_{z}^{\mathrm{d}}$ est solution d'un problème du type $\mathbf{H} / /$. On trouve que si $y>\max f(x)$ :

$$
\begin{aligned}
H_{z}^{\mathrm{d}}=\sum_{n=-\infty}^{+\infty} \sqrt{\frac{\varepsilon_{0}}{\mu_{0}}} & \left(\alpha a_{y}+\beta a_{x}\right) \times \\
& \times B_{n}^{2} \exp \left(i \tilde{k}\left(\tilde{\alpha}_{n} x+\tilde{\beta}_{n} y\right)\right),
\end{aligned}
$$

les coefficients $B_{n}^{2}$ étant obtenus par le programme $H / /$ à partir de $\tilde{k}$ et $\tilde{\alpha}$.

$E_{z}$ et $H_{z}$ étant ainsi déterminés, les autres composantes du champ électromagnétique s'obtiennent aisément par des formules très connues dans la théorie des guides d'onde [31] et résultant directement des équations de Maxwell :

$$
\begin{aligned}
& \tilde{k}^{2} H_{x}=-i \omega \varepsilon_{0} \frac{\partial E_{z}}{\partial y}+i k \gamma \frac{\partial H_{z}}{\partial x} \\
& \tilde{k}^{2} H_{y}=i \omega \varepsilon_{0} \frac{\partial E_{z}}{\partial x}+i k \gamma \frac{\partial H_{z}}{\partial y} \\
& \tilde{k}^{2} E_{x}=i \omega \mu_{0} \frac{\partial H_{z}}{\partial y}+i k \gamma \frac{\partial E_{z}}{\partial x} \\
& \tilde{k}^{2} E_{y}=-i \omega \mu_{0} \frac{\partial H_{z}}{\partial x}+i k \gamma \frac{\partial E_{z}}{\partial y} .
\end{aligned}
$$

Tous calculs faits, on trouve que si $y>\max f(x)$ :

$$
\begin{aligned}
\mathbf{E}_{\mathrm{d}} & =\sum \mathbf{E}_{n}^{\mathrm{d}}(x, y) \\
& =\sum_{n} \exp \left[i \tilde{k}\left(\tilde{\alpha}_{n} x+\tilde{\beta}_{n} y\right)\right]\left(P_{n} \mathbf{e}_{1}+Q_{n} \mathbf{e}_{2}+R_{n} \mathbf{e}_{3}\right),
\end{aligned}
$$

avec

$$
\begin{aligned}
& P_{n}=-\frac{1}{\sqrt{1-\gamma^{2}}}\left[\left(\alpha a_{y}+\beta a_{x}\right) \tilde{\beta}_{n} B_{n}^{2}-\gamma a_{z} \tilde{\alpha}_{n} B_{n}^{1}\right], \\
& Q_{n}=\frac{1}{\sqrt{1-\gamma^{2}}}\left[\left(\alpha a_{y}+\beta a_{x}\right) \tilde{\alpha}_{n} B_{n}^{2}-\gamma a_{z} \tilde{\beta}_{n} B_{n}^{1}\right], \\
& R_{n}=a_{z} B_{n}^{1},
\end{aligned}
$$

tandis que les considérations habituelles sur le vecteur de Poynting montrent que l'efficacité $\rho_{n}$ dans l'ordre $n$ est donnée par :

$$
\rho_{n}=\frac{\beta_{n}}{\beta}\left[\left|B_{n}^{2}\right|^{2}\left(\tilde{\alpha} a_{y}+\tilde{\beta} a_{x}\right)^{2}+\left|B_{n}^{1}\right|^{2} \frac{a_{z}^{2}}{1-\gamma^{2}}\right] .
$$

Finalement, les formules (23) et (27) prouvent que si $y>\max f(x)$ le champ est donné par le développement de Rayleigh :

$$
\begin{aligned}
\boldsymbol{E}^{\mathrm{d}}(x, y, z)=\sum_{n} & \left(P_{n} \mathbf{e}_{1}+Q_{n} \mathbf{e}_{2}+R_{n} \mathbf{e}_{3}\right) \times \\
& \times \exp \left(i k\left(\alpha_{n} x+\beta_{n} y+\gamma z\right)\right)
\end{aligned}
$$

où

$$
\alpha_{n}=\alpha+n \frac{\lambda}{d}=\tilde{\alpha}_{n} \sqrt{1-\gamma^{2}}
$$

et

$$
\beta_{n}=\sqrt{1-\gamma^{2}-\alpha_{n}^{2}}=\tilde{\beta}_{n} \sqrt{1-\gamma^{2}} .
$$

On peut 'vérifier que si $\left|\alpha_{n}\right|$ est inférieur à l'unité, le terme correspondant représente une onde plane se propageant suivant la direction $\mathbf{u}_{n}\left(\alpha_{n}, \beta_{n}, \gamma\right)$. Quel que soit $n$, cette direction fait avec $O z$ le même angle que la direction incidente $\mathbf{u}\left(\mathbf{u} . \mathbf{e}_{3}=\mathbf{u}_{n} \cdot \mathbf{e}_{3}\right)$. Les différents vecteurs d'ondes diffractés se répartissent donc sur un cône d'axe $O z$ et on parle de diffraction conique. La formule (28) montre que le calcul des efficacités en diffraction conique découle immédiatement des calculs effectués pour chacun des deux cas traités aux paragraphes I. 1 et I. 3 et qualifiés pour cela de fondamentaux. Les formules (30) jouent le même rôle que la formule des réseaux (16) dans le cas classique $(\gamma=0)$ et suggèrent une construction simple des vecteurs $\mathbf{u}_{n}$ à partir du vecteur unitaire $\mathbf{u}(\alpha,-\beta, \gamma)$ associé à la propagation de l'onde incidente. Cette construction est indiquée sur la figure 3 . Soit $\delta$ une sphère de centre $\mathrm{O}$ et de rayon unité et $A$ l'intersection de cette sphère avec son diamètre parallèle à $\mathbf{u}(\mathbf{O A}=\mathbf{u})$. Le plan $\pi$ passant par $A$ et perpendiculaire à $O z$ coupe $S$ suivant un cercle $\mathrm{C}$. Si $A_{0}, A_{1}, \ldots, A_{j}$ sont les intersections avec $\mathcal{C}$ d'une famille de demi-droites parallèles à $O y$ et équidistantes de $\lambda / d$, on vérifie d'après (30) que $\mathbf{O A}_{0}, \mathbf{O A}_{1}, \ldots, \mathbf{O A}_{j}$ sont respectivement égaux à $\mathbf{u}_{0}, \mathbf{u}_{1}, \ldots, \mathbf{u}_{j}$. Tous les $\mathbf{O} \mathbf{A}_{j}$ sont sur le cône circulaire de sommet $\mathrm{O}$ et de directrice $\mathrm{C}$. Ce cône dégénère en un plan si $\gamma=0$; c'est alors un grand cercle de $S$ et l'on retrouve la construction classique [6].

Examinons maintenant un cas particulier dont on reparlera dans les applications. Supposons que le vecteur d'onde incident $\mathbf{k}$ varie de sorte que sa projection sur le plan de section principale du réseau (plan $x O y$ ) soit fixe : $\tilde{\alpha}, \tilde{\beta}, \tilde{k}, \tilde{\alpha}_{n}, \tilde{\beta}_{n}$ restent constants et $\mathbf{k}$ reste orthogonal à un vecteur unitaire $\mathbf{U}(\tilde{\beta}, \tilde{\alpha}, 0) \mathrm{du}$ plan $x O y$. Supposons de plus que l'état de polarisation de l'onde incidente soit repéré par la donnée de l'angle $\delta$ des vecteurs $\mathbf{U}$ et $\mathbf{a}$. 


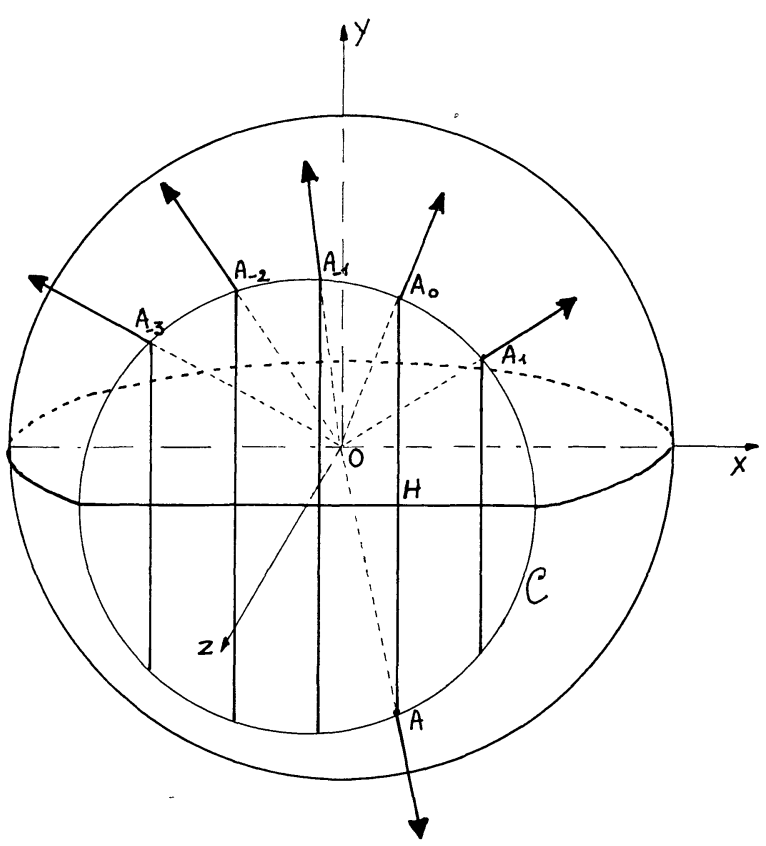

FIG. 3. - Diffraction conique : la sphère a un rayon unité, l'onde incidente se propage suivant $\mathrm{OA}$, toutes les parallèles à $A H$ sont équidistantes de $\lambda / d$.

Alors :

$$
\begin{aligned}
& \text { a. } \mathbf{U}=\cos \delta=a_{x} \tilde{\beta}+a_{y} \tilde{\alpha}, \\
& \text { a.u }=0=a_{x} \alpha-a_{y} \beta+a_{z} \gamma \text {, } \\
& \frac{-a_{z} \gamma}{\sqrt{1-\gamma^{2}}}=a_{x} \tilde{\alpha}-a_{y} \tilde{\beta},
\end{aligned}
$$

et, par combinaison :

$$
\begin{aligned}
& 1-a_{z}^{2}=a_{x}^{2}+a_{y}^{2}+\frac{a_{z}^{2} \gamma^{2}}{1-\gamma^{2}}, \\
& \frac{a_{z}^{2}}{1-\gamma^{2}}=\sin ^{2} \delta .
\end{aligned}
$$

Donc, l'efficacité $\rho_{n}$ reste constante pour un état de polarisation fixé puisque, d'après (28) :

$$
\rho_{n}=\frac{\tilde{\beta}_{n}}{\beta}\left(\left|B_{n}^{2}\right|^{2} \cos ^{2} \delta+\left|B_{n}^{1}\right|^{2} \sin ^{2} \delta\right) .
$$

Elle est extrémale pour $\delta=0$ et $\delta=\pi / 2$ et ces deux valeurs définissent deux états de polarisation remarquables notés $\boldsymbol{E} \perp$ ( $\boldsymbol{E}$ perpendiculaire aux sillons) et $\mathfrak{H} \perp$ ( $\mathfrak{H}$ perpendiculaire aux sillons) qui généralisent les cas respectivement notés $\mathbf{H} / /$ et $\mathbf{E} / /$ dans les montages classiques $(\gamma=0)$.

Résumons les résultats obtenus: En «diffraction conique ", les problèmes géométriques peuvent être rapidement résolus par une construction simple (Fig. 3) et grâce à la formule (28) les problèmes énergétiques ne nécessitent pas l'élaboration de programmes nouveaux.

Enfin, si l'onde incidente varie de sorte que la pro- jection de son vecteur d'onde sur le plan de section principale du réseau soit fixe :

a) la projection sur ce même plan du vecteur d'onde diffracté dans l'ordre $n$ reste également fixe,

b) l'efficacité dans l'ordre $n$ ne varie pas non plus pourvu que l'état de polarisation de l'onde incidente soit fixé.

I. 7 Conclusion. - Le formalisme intégral tel que nous venons de le décrire permet de traiter tous les profils qui peuvent être décrits par la donnée d'une fonction $y=f(x)$. C'est le cas de la majorité des réseaux ayant un intérêt pratique. Le réseau créneau (Fig. 4a) fait cependant exception. Mais sa géométrie très particulière permet un traitement spécial car, à l'intérieur de ses sillons, la forme du champ est du même type que celle que l'on observe dans un guide rectangulaire. L'existence de cette forme modale est à l'origine des importants travaux de Wirgin ([24], [32] à à [35]) et d'une de nos récentes publications [36]. L'idée consiste à raccorder des fonctions continues judicieusement choisies qui s'expriment par un développement de Rayleigh ou une forme modale suivant que l'on est à l'extérieur ou à l'intérieur des sillons. Les méthodes semi-analytiques ainsi mises au point s'appliquent aussi au réseau de tiges à section rectangulaire (Fig. 4b).

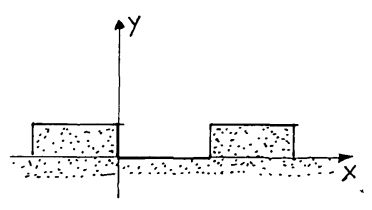

$a$

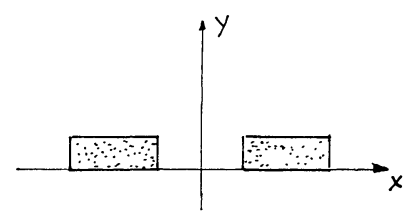

FIG. 4. - 4a. Réseau créneau.

$4 b$. Réseau de tiges à sections rectangulaires.

II. Le formalisme différentiel. - Le traitement numérique des systèmes différentiels ayant été très étudié, on peut essayer de ramener le problème du réseau à l'étude d'un tel système. Les deux exemples qui suivent vont montrer que cette nouvelle approche est souvent compétitive avec la technique des équations intégrales.

II. 1 ETUde DU RÉSEAU PAR TRANSMISSION [37], [38]. - Supposons la perméabilité relative $\mu$ constante dans tout l'espace et soit $a$ un réel positif. Soient $D_{1}$ et $\mathrm{D}_{2}$ les domaines respectivement définis par $y>a$ et $y<0$ à l'intérieur desquels la permittivité relative est constante et respectivement égale à $\varepsilon_{1}$ et $\varepsilon_{2}$. D'une façon très générale, nous dirons que le domaine $\mathfrak{D}$ $(0<y<a)$ est un réseau par transmission de pas $d=2 \pi / K$ si, dans $\mathcal{D}$ :

a) $\varepsilon$ est indépendant de $z$,

b) pour tout $y: \varepsilon(x+d, y)=\varepsilon(x, y)$,

c) $\varepsilon(x, y)$ est constant par région. 
Les figures 5 illustrent cette définition sur quelques exemples d'intérêt pratique.

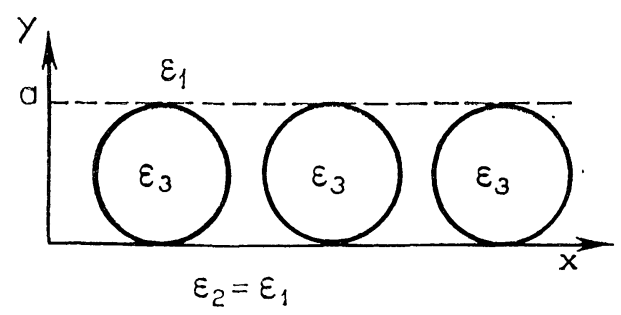

FIG. $5 a$.

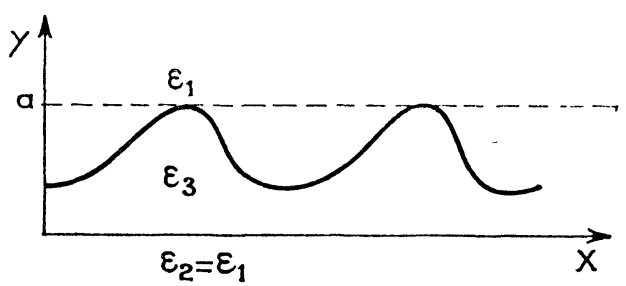

FIG. $5 b$.

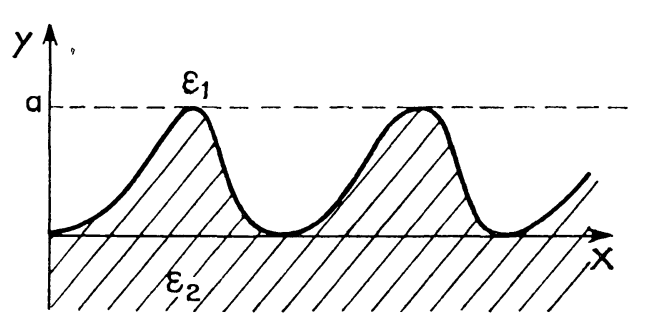

FIG. $5 c$.

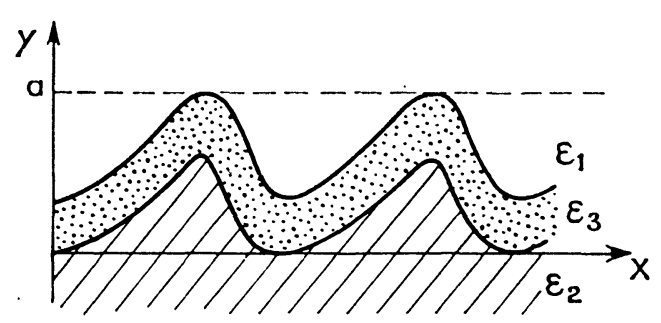

FIG. $5 d$.

FIG. 5. - Profils de réseaux par transmission.

$5 a$. Réseau de tiges cylindriques.

$5 b$. Réseau transparent de permittivité $\varepsilon_{3}$.

$5 c$. Réseau tracé sur un matériau de permittivité $\varepsilon_{2}$.

$5 d$. Le matériau de permittivité $\varepsilon_{2}$ est recouvert d'une couche de permittivité $\varepsilon_{3}$.

Plaçons-nous, pour simplifier l'exposé, dans le cas classique $(\gamma=0)$ en incidence normale et supposons que $\mathbf{E}$ soit parallèle aux traits $(\mathbf{E} / /)$ pour pouvoir utiliser les notations du paragraphe 1 , première partie. Les équations de Maxwell conduisent à l'équation aux dérivées partielles :

$$
\Delta E+k^{2}(x, y) E=0,
$$

où $k^{2}(x, y)$ est la fonction, constante par région, périodique en $x$, et égale à $\varepsilon \mu \omega^{2} / c^{2}$. Cette équation est vraie au sens des distributions car $E$ est une fonction continue (conservation de la composante tangentielle de $\mathbf{E}$ ) à dérivée normale continue (conservation de la composante tangentielle de $\mathbf{H}$ ). L'unicité de la solution implique encore la périodicité de $E$ en $x$. On peut donc poser :

$$
\begin{aligned}
& E(x, y)=\sum_{n=-\infty}^{+\infty} E_{n}(y) \exp (i n K x) \\
& k^{2}(x, y)=\sum_{n=-\infty}^{+\infty} C_{n}(y) \exp (i n K x)
\end{aligned}
$$

avec :

$$
C_{n}(y)=\frac{1}{d} \int_{0}^{\mathrm{d}} k^{2}(x, y) \exp (-i n K x) \mathrm{d} x .
$$

Nous savons que, compte tenu d'une condition d'ondes sortantes pour $y \rightarrow \pm \infty$, (32) conduit à un développement de Rayleigh dans $\mathscr{D}_{1}$ et $\mathscr{D}_{2}$, régions dans chacune desquelles $k^{2}$ est une constante :

Si $y \geqslant a$ :

$$
\begin{aligned}
E(x, y)=\exp ( & \left.-i k_{1} y\right)+ \\
& +\sum_{n=-\infty}^{+\infty} R_{n} \exp \left(i k_{1}\left(\alpha_{n} x+\beta_{n} y\right)\right),
\end{aligned}
$$

avec

$$
k_{1}=\frac{\omega}{c} \sqrt{\varepsilon_{1} \mu}, \quad \alpha_{n}=n \frac{K}{k_{1}}, \quad \beta_{n}=\sqrt{1-\alpha_{n}^{2}} .
$$

Si $y \leqslant 0$ :

$$
E(x, y)=\sum_{n=-\infty}^{+\infty} T_{n} \exp \left(i k_{2}\left(\alpha_{n} x-\beta_{n} y\right)\right)
$$

avec

$$
k^{2}=\omega^{2} \varepsilon_{2} \mu .
$$

On vérifie que l'identification des coefficients de Fourier des deux membres de (31) conduit dans $\mathcal{D}$ à :

$$
\frac{\mathrm{d}^{2} E_{n}}{\mathrm{~d} y^{2}}-n^{2} K^{2} E_{n}+\sum_{m=-\infty}^{+\infty} C_{n-m} E_{m}=0
$$

c'est-à-dire à un système différentiel pour les fonctions inconnues $E_{n}(y)$. Ces fonctions définies sur $(0, a)$ satisfont de plus des conditions aux limites que l'on déduit immédiatement de (34) et (35) :

$\frac{\mathrm{d} E_{0}}{\mathrm{~d} y}(a)-i k_{1} E_{0}(a)=-2 i k_{1} \exp \left(-i k_{1} a\right)$,

$\frac{\mathrm{d} E_{n}}{\mathrm{~d} y}(a)-i k_{1} \beta_{n} E_{n}(a)=0$ si $n \neq 0$,

$\frac{\mathrm{d} E_{n}}{\mathrm{~d} y}(0)+i k_{2} \beta_{n} E_{n}(0)=0$.

Ainsi, en admettant que $E$ est convenablement décrit par $N$ termes de son développement en série de Fourier, nous devons résoudre un système de $N$ équa- 
tions différentielles linéaires que l'on peut écrire sous forme matricielle :

$$
\begin{aligned}
& \frac{\mathrm{d}^{2} \mathcal{E}}{\mathrm{d} y^{2}}=\mathcal{A}(y) \varepsilon(y), \\
& \frac{\mathrm{d} \delta}{\mathrm{d} y}(0)+\mathfrak{L}_{0} \mathcal{E}(0)=0, \\
& \frac{\mathrm{d} \mathcal{E}}{\mathrm{d} y}(a)+\mathfrak{L}_{a} \mathcal{E}(a)=\mathcal{G},
\end{aligned}
$$

où $\delta$ est une matrice colonne inconnue d'élément $E_{n}(y)$, $\mathcal{A}$ une matrice carrée connue,

$\mathfrak{L}_{0}, \mathfrak{L}_{a}$ et $\mathbf{g}$ des matrices diagonales ou colonnes connues introduites pour traduire (37) et (38).

La description des techniques utilisées pour la résolution de ce système est faite dans les publications originales [37], [38], [40]. On obtient rapidement de bons résultats pour les réseaux diélectriques $(\varepsilon(x, y)$ réel), mais on rencontre de sérieuses difficultés pour les réseaux conducteurs ( $\varepsilon$ complexe à partie imaginaire élevée). Cet échec partiel est sans doute lié à des difficultés purement numériques qui apparaissent aussi dans le formalisme intégral.

II. 2 ETUde DU RÉSEAU PARFAITEMENT CONDUCTEUR, UTILISATION DES TRANSFORMATIONS CONFORMES. D'après le paragraphe I. 6 de la première partie, nous pouvons nous limiter aux deux cas fondamentaux de polarisation $\mathbf{E} / /$ et $\mathbf{H} / /$. Les notations restent les mêmes que pour le formalisme intégral à un petit changement près que nous croyons utile pour faciliter la tâche du lecteur qui voudrait se reporter aux publications originales [39], [40], [29]. Dans ce paragraphe, l'équation de la surface diffractante est $y=g(x)$ au lieu de $y=f(x)$ et le pas $d$ du réseau est systématiquement égal à $2 \pi$. Suivant que le vecteur champ électrique est parallèle aux sillons (cas $\mathbf{E} / /$ ) ou dans le plán $x O y$ (cas $\mathbf{H} / /)$, nous prenons respectivement comme fonction inconnue $f(x, y)$ la composante sur $O z$ du champ électrique total ou la composante sur ce même axe de l'excitation magnétique totale. On sait que cette fonction vérifie :

$$
\begin{gathered}
\Delta f+k^{2} f=0 \text { pour } y>g(x), \\
f(x+2 \pi, y)=f(x, y) \exp (2 i \pi k \sin \theta), \\
f_{r}-f_{\text {incident }} \sim \sum_{n=-\infty}^{+\infty} B_{n} \exp \left(i k \alpha_{n} x+i k \beta_{n} y\right) \\
\text { pour } y \rightarrow+\infty, \\
f(x, g(x))=0 \quad(\operatorname{cas} \mathbf{E} / /)
\end{gathered}
$$

ou

$$
\frac{\mathrm{d} f}{\mathrm{~d} n}(x, g(x))=0 \quad(\operatorname{cas} \mathbf{H} / /),
$$

les coefficients $\alpha_{n}$ et $\beta_{n}$ qui figurent dans le développe- ment de Rayleigh (41) ayant leur habituelle signification :

$$
\alpha_{n}=\sin \theta+n \frac{\lambda}{2 \pi}, \quad \beta_{n}^{2}=1-\alpha_{n}^{2} .
$$

La détermination de $f$ est un problème aux dérivées partielles caractérisé par une équation simple (équation de Helmholtz) et une condition aux limites rendue peu sympathique par la nature géométrique de la surface sur laquelle on l'écrit (la surface du réseau). $\mathrm{Ne}$ serait-il pas plus agréable, même au prix d'une légère complication de l'équation aux dérivées partielles, d'avoir à écrire la condition aux limites pour une surface sur laquelle une des coordonnées reste constante ? C'est là l'idée fondamentale de cette nouvelle formulation (Fig. 6) dans laquelle on recherche

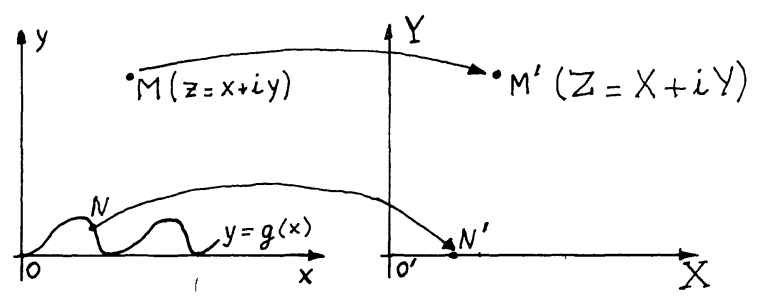

FIG. 6. - La transformation conforme : à tout point $M$ du plan $x O y$, on associe un point $M^{\prime}$ du plan $X O^{\prime} Y$ de sorte qu'un point $N$ situé sur le réseau corresponde à un point $N^{\prime}$ de l'axe $O^{\prime} X$.

une transformation ponctuelle qui transforme le réseau en un miroir plan sans trop perturber (39), (40) et (41).

On peut vérifier que, si nous posons $z=x+i y$ et $Z=X+i Y$, la famille de transformations conformes définies par :

$$
z=Z+\sum_{n=0}^{\infty} \varepsilon_{n} \exp (i n Z) \text { avec } \varepsilon_{n}=a_{n}+i b_{n}
$$

- respecte la condition d'ondes sortantes (41) car

$Z \sim z$ quand $Y \rightarrow+\infty$;

- respecte la périodicité (40);

- remplace (34) par :

$$
\frac{\partial^{2} F}{\partial X^{2}}+\frac{\partial^{2} F}{\partial Y^{2}}+K^{2}(X, Y) F(X, Y)=0\left(^{7}\right)
$$

avec

$$
K^{2}(X, Y)=k^{2}|\mathrm{~d} z / \mathrm{d} Z|^{2},
$$

- remplace (42) par:

$$
F(X, 0)=0 \text { pour le cas } \mathbf{E} / /
$$

et

$$
\frac{\partial F}{\partial Y}(X, 0)=0 \text { pour le cas } \mathbf{H} / /,
$$

(7) On ne confondra pas $K(X, Y)$ avec la pulsation spatiale systématiquement utilisée dans les autres paragraphes. 
- transforme l'axe $O X$ en la courbe d'équations paramétriques :

$$
\left.\begin{array}{l}
x=X+\sum_{n=0}^{\infty}\left(a_{n} \cos n X-b_{n} \sin n X\right) \\
y=\sum_{n=0}^{\infty}\left(a_{n} \sin n X+b_{n} \cos n X\right)
\end{array}\right\}
$$

courbe qui peut être précisément le profil du réseau si les coefficients réels $a_{n}$ et $b_{n}$ sont bien choisis.

Il se trouve que le coefficient $K^{2}(X, Y)$ est périodique en $X$ (de période $2 \pi$ ) et tend exponentiellement vers $k^{2}$ si $Y$ tend vers l'infini. Ainsi, si l'on confond $K^{2}$ avec $k^{2}$ dès que $Y$ devient supérieur à un nombre $A$ que l'on détermine par quelques tests numériques, nous retombons sur un problème du meme type qu'au paragraphe précédent.

Trouver la fonction $F(X, Y)$ telle que :

$\frac{\partial^{2} F}{\partial X^{2}}+\frac{\partial^{2} F}{\partial Y^{2}}+K^{2}(X, Y) F=0 \quad$ pour $\quad 0<Y \leqslant A$

$F(X+2 \pi, Y)=F(X, Y) \exp (i 2 \pi k \sin \theta) \quad \forall Y$,

$F-F_{\text {incident }}=\sum_{n=-\infty}^{+\infty} B_{n} \exp \left(i k \alpha_{n} X+i k \beta_{n} Y\right)$

pour $Y \geqslant A$.

$$
F(x, 0)=0, \quad \text { ou } \quad \frac{\mathrm{d} F}{\mathrm{~d} Y}(X, 0)=0 .
$$

Il suffit de poser :

$$
\begin{aligned}
F(X, Y) & =\sum_{n=-\infty}^{+\infty} F_{n}(Y) \exp \left(i \alpha_{n} X\right), \\
C_{n}(Y) & =\frac{1}{2 \pi} \int_{0}^{2 \pi}\left|\frac{\mathrm{d} z}{\mathrm{~d} Z}\right|^{2} \exp (-i n X) \mathrm{d} X,
\end{aligned}
$$

et d'expliciter (45) pour trouver que les fonctions $F_{n}(Y)$ sont solutions du système infini d'équations différentielles couplées :

$$
\frac{\mathrm{d}^{2} F_{n}}{\mathrm{~d} Y^{2}}-k^{2} \alpha_{n}^{2} F_{n}+k^{2} \sum_{m=-\infty}^{+\infty} C_{n-m}(Y) F_{m}=0 .
$$

$\mathrm{Si}$, comme toujours, nous admettons que $F(X, Y)$ est suffisamment bien décrit par $2 N+1$ termes du développement (48), le problème initial se ramène à la détermination de $2 N+1$ fonctions $F_{n}(Y)$ définies sur $(0, A)$ vérifiant :

$$
\frac{\mathrm{d}^{2} F_{n}}{\mathrm{~d} Y^{2}}=\sum_{m=-D}^{+N} v_{n m}(Y) F_{n}(Y)
$$

avec

$$
v_{n m}(Y)=-k^{2} C_{n-m}(Y)+k^{2} \alpha_{n}^{2} \delta_{n m},
$$

et satisfaisant les conditions aux limites :

$$
\begin{aligned}
& F_{n}(0)=0(\operatorname{cas} \mathbf{E} / /) \text { ou } \frac{\mathrm{d} F_{n}}{\mathrm{~d} Y}(0)=0(\operatorname{cas} \mathbf{H} / /) . \\
& F_{n}(A)=B_{n} \exp \left(i k \beta_{n} A\right)+\delta_{n_{0}} \exp \left(-i k \beta_{n} A\right) .
\end{aligned}
$$

La connaissance de $F_{n}(A)$ fournit immédiatement les coefficients $B_{n}$ permettant le calcul des efficacités.

L'intégration numérique de ce système différentiel par l'utilisation de l'algorithme de Cowell-Noumerov [40] a permis d'obtenir des temps de calculs encore plus faibles que ceux du formalisme intégral (de l'ordre de grandeur de la seconde par point sur UNIVAC 1108). Le problème délicat reste celui de l'obtention des coefficients $a_{n}$ et $b_{n}$ décrivant la transformation conforme susceptible d'associer l'axe $Y=0$ au profil d'un réseau donné. Il a été résolu analytiquement ou numériquement pour la plupart des profils usuels [40] et les résultats ainsi obtenus ont été confrontés avec succès à ceux du formalisme intégral. Ceci montre, au passage, qu'un miroir plan recouvert d'une couche diélectrique inhomogène, d'indice $n(X, Y)$ périodique en $X$, est parfois équivalent à un réseau échelette. L'effet de blaze n'est donc pas, comme on l'a cru longtemps, l'apanage du réseau échelette et il peut être produit par de nombreuses structures périodiques. La présence de "facettes jouant le rôle de miroirs » n'est pas du tout indispensable comme le laissaient croire les cours d'Optique.

III. Applications. - De nombreuses efficacités calculées par les différentes méthodes que nous venons de décrire ont été confrontées aux efficacités mesurées. Cette confrontation théorie-expérience qui a surtout été faite en ondes centimétriques en collaboration avec R. Deleuil ([41], [42], [43], [44]) atoujours été très satisfaisante. On peut donc se fier aux méthodes numériques pour prévoir les propriétés des réseaux et l'ordinateur est devenu dans ce domaine un collaborateur précieux voire indispensable.

III. 1 EfFICACITÉ DES RÉSEAUX ÉCHELETTES. - Si l'on suppose les facettes perpendiculaires entre elles, l'angle de blaze suffit pour caractériser la forme du profil. On peut donc, pour un montage donné, tracer rapidement les courbes d'efficacité. Ceci a été fait pour l'ordre -1 et pour les montages à déviation constante, dans la Nouvelle Revue d'Optique [45]. Les figures 7 donnent un aperçu des résultats. On y constate le très grand rôle de la polarisation et l'on devine le désarroi d'un utilisateur qui, dans un montage de Littrow (Fig. $7 b$ ) et pour $\theta=40^{\circ}$ mesurerait une efficacité égale à $40 \%$ (cas $\mathbf{E} / /$ ) ou $65 \%$ (lumière naturelle) de l'efficacité promise par les théories scalaires fixant à $40^{\circ}$ l'abscisse du maximum. On doit se souvenir que la formule simple $\lambda_{B}=2 d \sin \alpha$ permettant de prévoir la longueur d'onde de blaze $\lambda_{B}$ (pour laquelle l'efficacité est maximale) en fonction de l'angle de blaze $\alpha$ ne doit pas être utilisée lorsque $\lambda_{B} / d$ est voisin de l'unité [6], [45]. Notons enfin que le calcul numérique permet de prévoir l'influence sur l'efficacité d'un éventuel défaut de tracé [6] et de choisir le réseau à employer pour résoudre un problème spectroscopique donné [46].

III.2 EFFICACITÉ DES RÉSEAUX HOLOGRAPHIQUES 
PLANS. - On sait [47] que ceux-ci sont obtenus en enregistrant un phénomène d'interférence sur un support plan enduit d'une résine photopolymérisable. Après révélation et métallisation, on obtient un réseau réfléchissant dont le profil mal connu ne peut être déterminé qu'après la mise en œuvre de diverses techniques de microscopie électronique. En adoptant un modèle mathématique simple pour représenter les clichés obtenus, on peut se livrer aux mêmes calculs que pour les échelettes, mais le travail est beaucoup moins avancé car le profil dépend ici de plusieurs paramètres. On peut cependant énoncer les résultats suivants ([48], [49]) : L' « effet de blaze » existe et peut être très important; la «longueur d'onde de blaze » dépend beaucoup du profil et semble augmenter avec la profondeur des sillons ; il en est de même de l'efficacité maximale qui est par ailleurs très sensible à la polarisation ; pour

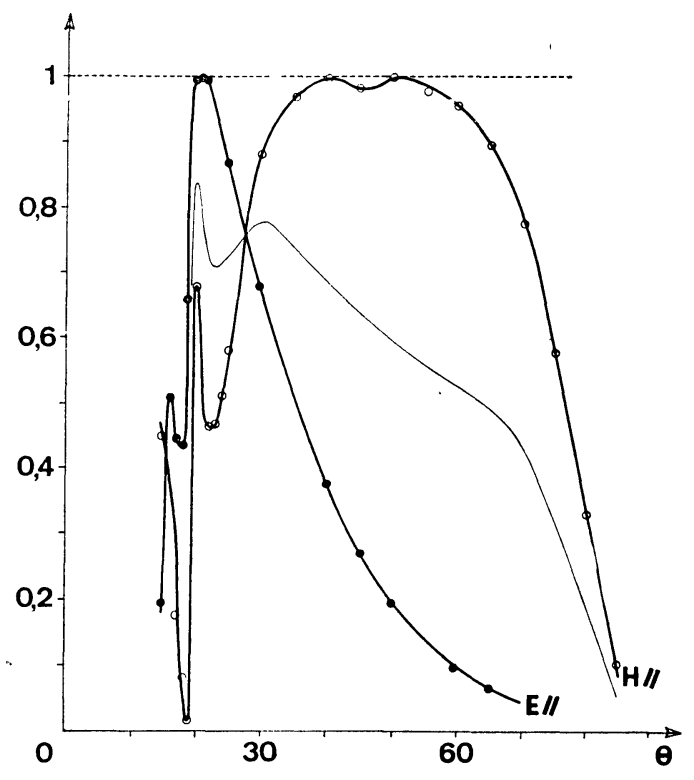

FIG. $7 a$.

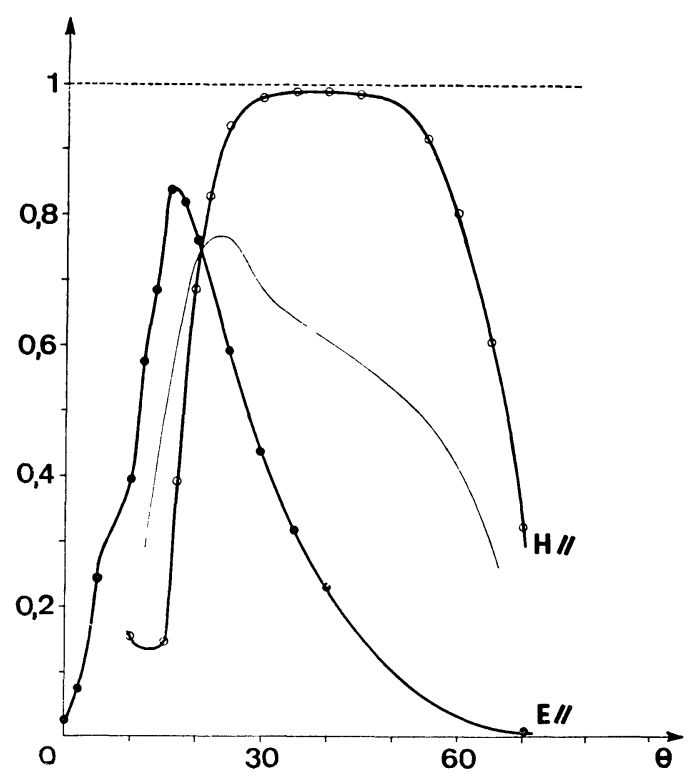

FIG. $7 b$.

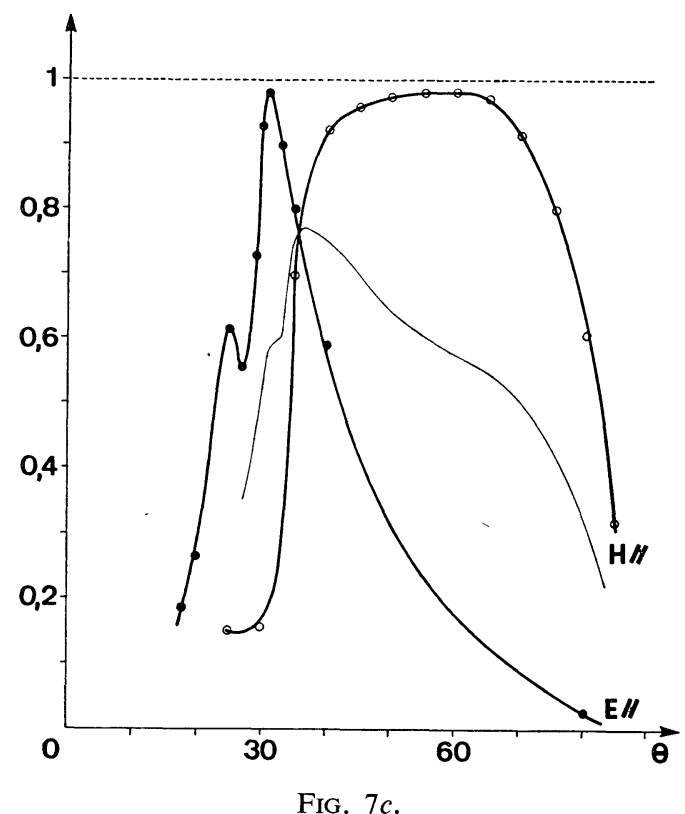

FIG. 7. - Efficacité des réseaux échelettes : chaque graphique donne l'efficacité dans l'ordre - 1 en fonction de l'angle d'incidence $\theta$ pour une déviation $\mathrm{D}$ et un «angle de blaze» de $40^{\circ}$

7a. $\mathrm{D}=0$.

7b. $\mathrm{D}=+15$.

7c. $\mathrm{D}=-15$.

la polarisation $\mathbf{H} / /$ les courbes d'efficacité sont très irrégulières par suite d'une grande sensibilité aux anomalies de Wood. Les figures 8 correspondent aux

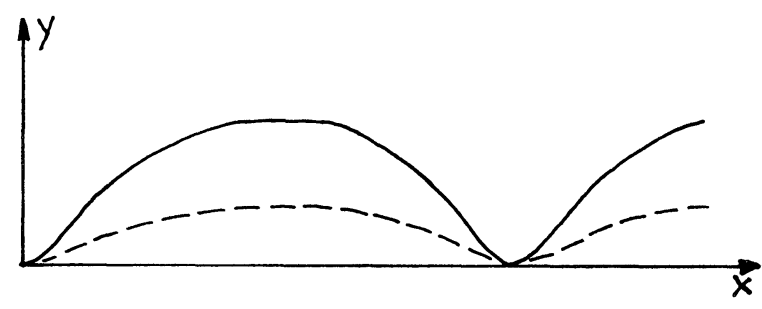

FIG. $8 a$.

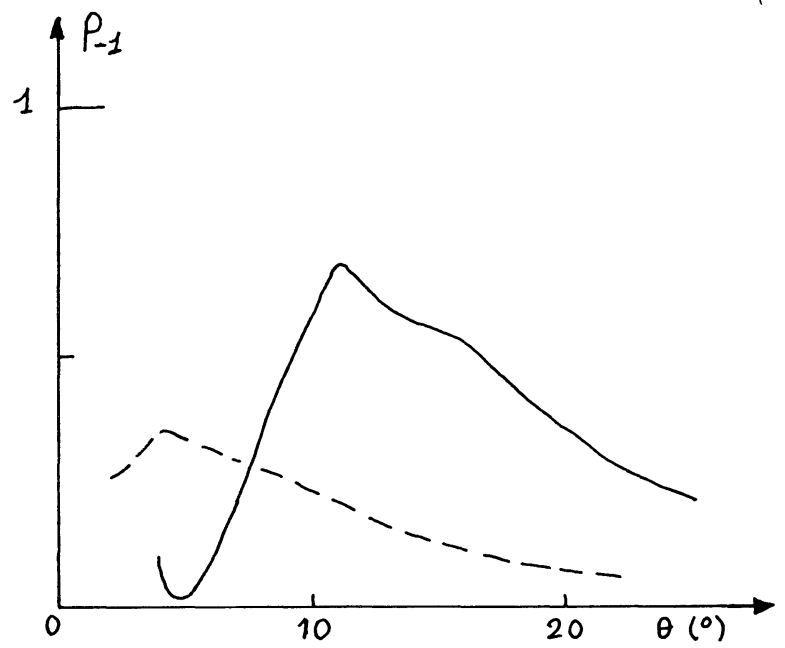

FIG. $8 b$. 


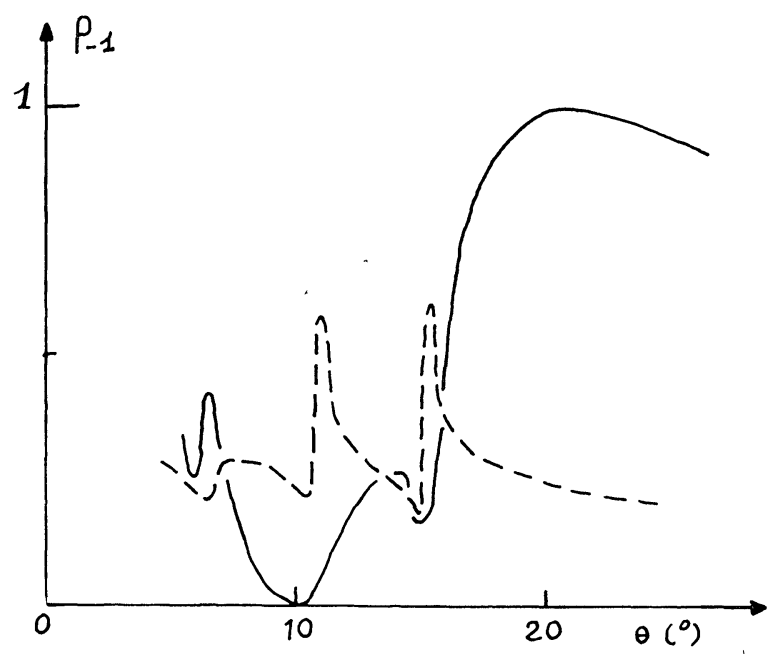

FIG. $8 c$.

FIG. 8. - Efficacité des réseaux holographiques. On donne les efficacités en Littrow et dans l'ordre - 1 pour les deux profils décrits sur la figure $8 a$ et pour les deux cas fondamentaux de polarisation $\mathbf{E} / /$ (Fig. 8b) et $\mathbf{H} / /$ (Fig. 8c).

premiers résultats obtenus lors d'une étude que nous poursuivons actuellement en collaboration avec JobinYvon.

III.3 PouvoIr RÉFLECTEUR A INCIDENCE FIXE. On trouve dans [6], [49] et [50] des courbes donnant pour une incidence $\theta$ fixée l'efficacité dans l'ordre zéro. Il faut retenir que si $\mathbf{H}$ est parallèle aux traits, ces courbes sont très voisines d'une "marche d'escalier » (Fig. 9). Cette particularité a été mise à profit par

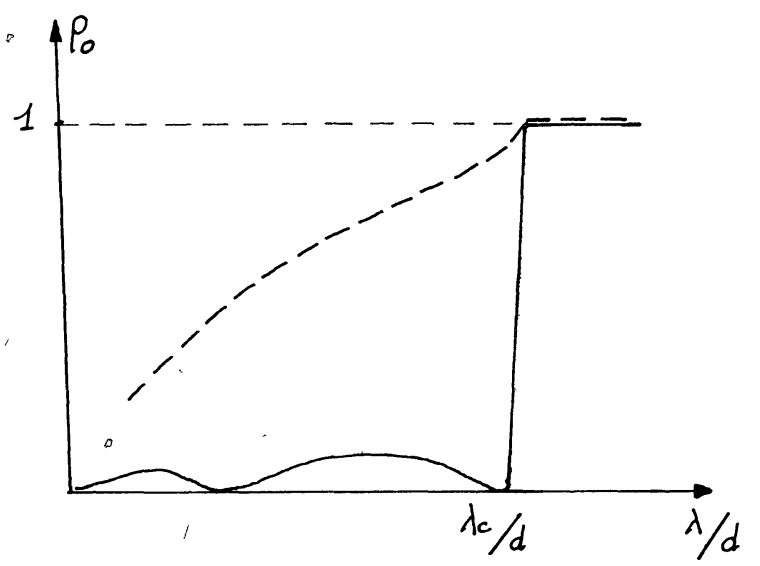

FIG. 9. - Allure de l'efficacité d'un réseau dans l'ordre 0 , pour un angle d'incidence $\theta$ fixé, dans le cas $\mathbf{H} / /$ (trait plein) ou $\mathbf{E} / /$ (trait interrompu).

A. Hadni et ses collaborateurs [51] pour réaliser des filtres de longueur d'onde (passe-haut) pour l'infrarouge lointain; le calcul permet de rechercher la meilleure valeur de $\theta$ et la forme optimale des sillons.

III. 4 CONSÉQUENCES DU THÉORÈME DE RÉCIPROCITÉ ; FILTRES DE LONGUEUR D'ONDE. - On peut, pour les réseaux, énoncer le théorème de réciprocité suivant

dont on trouvera une démonstration simple dans [52] :

Si $\mathbf{k}^{\mathbf{d}}$ est le vecteur d'onde de l'une des ondes diffractées par un réseau éclairé par une onde plane de vecteur d'onde $\mathbf{k}$, le vecteur - $\mathbf{k}$ est associé à l'une des ondes diffractées par le réseau quand il est éclairé par une onde incidente de vecteur $-\mathbf{k}^{\mathbf{d}}$ et l'efficacité est la même dans les deux cas ; elle est indépendante du sens de propagation de la lumière.

On en déduit un corollaire important établi directement dans [50] :

L'efficacité $\rho_{0}$ dans l'ordre zéro est insensible à une rotation du réseau de $180^{\circ}$ autour d'un axe perpendiculaire à son plan moyen. Ceci explique que, comme l'avait constaté A. Hadni dans l'infrarouge lointain, l'efficacité $\rho_{-1}$ du montage de Littrow dans l'ordre - 1 soit la même dans l'une ou l'autre des deux configurations de la figure 10 pourvu que $\lambda / d>2 / 3$. Dans ces conditions en effet [50], seuls existent les ordres 0 et -1 , la conservation de l'énergie impose $\rho_{0}+\rho_{-1}=1$ et comme $\rho_{0}$ est insensible au retournement il en est de même de $\rho_{-1}$.

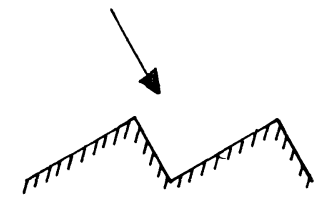

a)

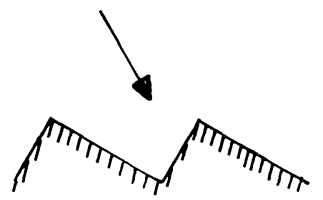

b)

FIG. 10. - On attaque le réseau sur sa grande facette (10a) ou sur sa petite facette $(10 b)$.

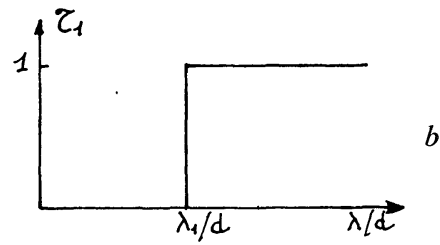

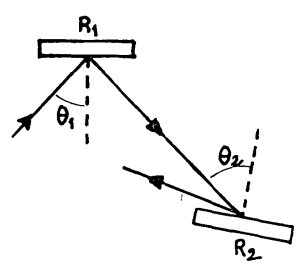

$a$

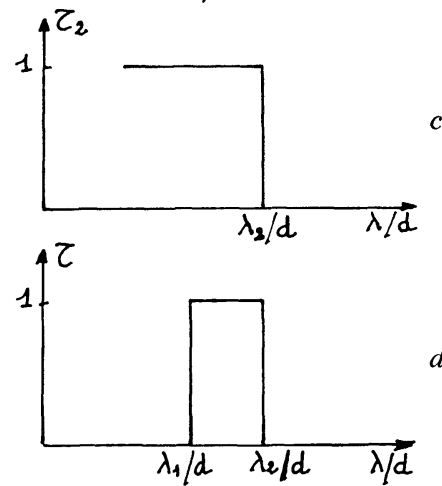

FIG. 11. - Filtres de longueur d'onde.

11a. Schéma de principe.

11b. Dans l'ordre $0, \mathrm{R}_{1}$ fonctionne en filtre passe-haut ;

$$
\lambda_{1} / d=1+\sin \theta_{1} .
$$

11c. Dans l'ordre $-1, \mathrm{R}_{2}$ fonctionne comme un filtre passe-bas car seuls existent les ordres 0 et -1 si

$$
\lambda_{2} / 2 d<\lambda / d<\lambda_{2} / d ; \lambda_{2} / d=1+\sin \theta_{2} .
$$

11d. L'ensemble $\mathrm{R}_{1}+\mathrm{R}_{2}$ constitue un filtre passe-bande; $\lambda_{1} / d=1+\sin \theta_{1}, \quad \lambda_{2} / d=1+\sin \theta_{2}$. 
Le théorème de réciprocité et les propriétés signalées au paragraphe précédent permettent de concevoir des filtres passe-bande en lumière polarisée. Soit en effet une onde plane rencontrant successivement (Fig. 11) deux réseaux $R_{1}$ et $R_{2}$ dont les traits sont parallèles à $\mathbf{H}$ et fonctionnant respectivement dans les ordres 0 et -1 . Si, pour simplifier, nous remplaçons la courbe en trait plein de la figure 9 par une «marche d'escalier », les figures $11 b, 11 c$ et $11 d$ donnent respectivement les transmissions $\mathfrak{C}_{1}, \mathfrak{C}_{2}$ et $\mathcal{C}$ de $\mathrm{R}_{1}, \mathrm{R}_{2}$ et de l'ensemble passe-bande $R_{1}+R_{2}$. La direction de l'onde transmise par ce filtre rudimentaire dépend malheureusement de $\lambda$, mais on montre [52] que le théorème de réciprocité permet de pallier facilement cet inconvénient par adjonction d'un troisième réseau convenablement disposé.

III. 5 SPECTROMÈTRE A TRANSMISSION ÍNDÉPENDANTE DE LA LONGUEUR D'ONDE. - Il s'agit d'une exploitation du résultat mis en valeur à la fin du paragraphe consacré à la diffraction conique. Le lecteur intéressé pourra se reporter à [53] pour une étude détaillée de cet instrument dont voici le principe (Fig. 12). Le réseau $R$ et le miroir $M$ sont solidaires de la plateforme $\mathrm{P}$ mobile autour de l'axe $\mathrm{OO}^{\prime}$. Le plan de $\mathrm{M}$ ainsi que celui des grandes facettes de $\mathrm{R}$ sont verticaux et ils font entre eux l'angle $\gamma$. Au foyer d'une lentille collimatrice $\mathrm{L}_{\mathrm{e}}$ fixe, d'axe optique $\mathbf{u}_{\mathrm{e}}$ horizontal et parallèle aux petites facettes de $R$ se trouve une source ponctuelle polychromatique $\mathrm{S}$. On montre qu'en faisant tourner la plateforme, on fait défiler les radiations correspondant aux différentes longueurs d'ondes sur

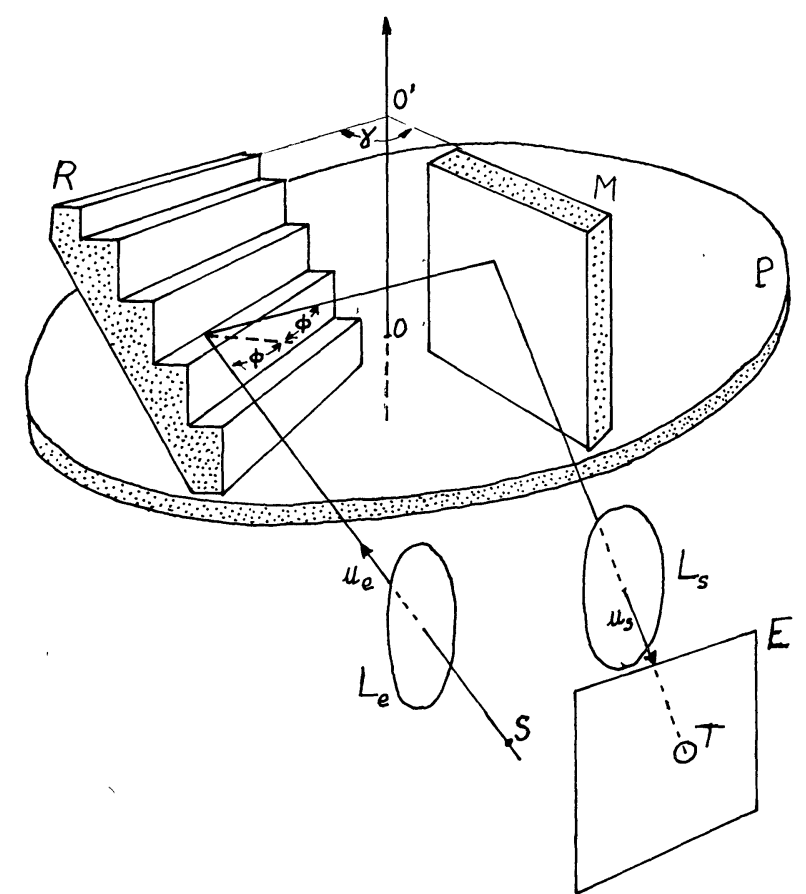

FIG. 12. - Principe d'un spectromètre à transmission indépendante de la longueur d'onde; la source $S$ est dans le plan focal objet de $\mathrm{L}_{e}$; $\mathrm{T}$ est un trou percé dans l'écran opaque $\mathrm{E}$ matérialisant le plan focal image de $L_{\mathbf{s}}$.

le foyer image d'une lentille fixe $\mathrm{L}_{s}$ dont l'axe optique $\mathbf{u}_{s}$ se déduit de $\mathbf{u}_{\mathrm{e}}$ par rotation de $2 \gamma$ autour de $\mathrm{OO}^{\prime}$. L'efficacité ou transmission de l'ensemble $\mathbf{R}+\mathbf{M}$ demeure constante et peut avoir une valeur théorique très voisine de l'unité quelle que soit la polarisation.

\section{Bibliographie}

[1] VAN DEN BERG (P. M.), Rigorous diffraction theory of optical reflection and transmission gratings, Thèse, 1971, Bronder-offset N. V., Rotterdam.

[2] Neureuther (A. R.) and ZAKI (K.), Alta frequenza, 1968,38 , special issue on URSI Symposium on electromagnetic waves.

[3] Schwartz (L.), Méthodes mathématiques pour les sciences physiques, Hermann, Paris, 1965.

[4] Bass (J.), Cours de Mathématiques, tome III, Masson, 1971.

[5] Pavageau (J.) et Bousquet (J.), Opt. Acta, 1970, 17, 469.

[6] Petit (R.), Rev. Opt., 1966, 249.

[7] Mc Clellan (R. P.) et Stroke (G. W.), J. Math. Phys., 1966, 45, 383.

[8] Lord Rayleigh, Proc. $R$. Soc., 1907, A, 79, 399.

[9] Fano (U.), J. Opt. Soc. Am., 1941, 31, 213.

[10] Meеcham (W. C.), J. Appl. Phys., 1956, 27, 361.

[11] Stroke (G. W.), Rev. Opt., 1960, 39, 350.

[12] Bousquet (P.), C. R. Acad. Sci., Paris, 1963, 256, 3422.

[13] Bousquet (P.), C. R. Acad. Sci., Paris, 1963, 257, 80.

[14] Petit (R.), Rev. Opt., 1963, 42, 263.

[15] Deleuil (R.), C. R. Acad. Sci., Paris, 1964, 258, 506.

[16] Yakovlev (E. A.), Opt. i Spektr., 1965, 19, 417.

[17] Janot (C.) et Hadni (A.), J. Physique, 1963, 24, 1073.

[18] Lippmann (B. A.), J. Opt. Soc. Am., 1953, 43, 408.

[19] Petit (R.) et Cadilhac(M.), C. R. Acad. Sci., Paris, 1966, Sér. A-B, 262, 468.
[20] Millar (R. F.), Proc. Camb. Phil. Soc., 1969, 65, 773.

[21] Millar (R. F.), Proc. Camb. Phil. Soc., 1971, 69, 217.

[22] Nevière (M.) et Cadilhac (M.), Opt. Commun., 1970, $2,235$.

[23] BARANTSEV (R. G.), Vestnik Leningradskogo Universiteta, 1965, 1, 66 .

[24] Wirgin (A.), Thèse, Paris, 1967, $\mathrm{n}^{\circ}$ CNRS : AO, 1429.

[25] Maystre (D.) et Petit (R.), C. R. Acad. Sci., Paris, 1970, Sér. B, 271, 400.

[26] Dumery (G.) et Filippi (P.), C. R. Acad. Sci., Paris, 1970, Sér. B, 270, 137-139.

[27] Uretsky (J. L.), Ann. Phys., 1965, 33, 400.

[28] Maystre (D.) et Petit (R.), Opt. Commun., 1971, 4, 97.

[29] Nevière (M.) et Cadilhac (M.), Opt. Commun., 1971, $4,13$.

[30] Bolomey (J. C.), Thèse, Paris, 1971, no CNRS : AO 5604.

[31] Goudet (G.) et Chavance (P.), Ondes centimétriques, Chiron, Paris, 73.

[32] Wirgin (A.), Alta frequenza, 1969, 38, 327.

[33] Wirgin (A.), Alta frequenza, 1969, 38, 332.

[34] Wirgin (A.), Nouv. Revue. Opt. Appl., 1970, 1, 161.

[35] Wirgin (A.), Nouv. Revue. Opt. Appl., 1970, 1, 165.

[36] Maystre (D.) et Petit (R.), Diffraction par un réseau lamellaire infiniment conducteur, Opt. Com., à paraître.

[37] Cerutti-Maori (G.), Petit (R.) et Cadilhac (M.), C. R. Acad. Sci., Paris, 1969, Sér. B, 268, 1060.

[38] Cerutti-Maori (G.), Thèse de $3^{\mathrm{e}}$ cycle, Paris, 1970. 
[39] Nevière (M.) et Cadilhac (M.), Opt. Commun., 1971, $3,379$.

[40] Nevière (M.), Cadilhac (M.) et Petit (R.), International symposium, Tbilissi, sept. 1971, Editions Nauka, Moscou.

[41] Petit (R.), Rev. Opt., 1966, 353-370.

[42] Deleuil (R.), Thèse, Marseille, 1969, no CNRS : AO 3601 .

[43] Deleuil (R.), Opt. Acta, 1969, 16, 23.

[44] Wirgin (A.) et Deleuil (R.), J.' Opt. Soc. Am., $1969,59,1348$

[45] Maystre (D.) et Petit (R.), Nouv. Revue. Opt. Appl., $1971,2,115$.

[46] Cordelle (J.), Laude (J. P.), Petit (R.), PieuChard (G.), Nouv. Revue. Opt. Appl., 1970, 1, 149.

[47] Labeyrie (A.) et Flamand (J.), Opt. Commun., 1969 , 1,5 .

[48] Maystre (D.) et Petit (R.), Opt. Commun., 1970, 2, 309.

[49] Maystre (D.) et Petit (R.), Opt. Commun., 1971, 4,25 .

[50] Petit (R.), Opt. Acta, 1967, 14, 301.
[51] Hadni (A.), Decamps (E.), Grandjean (D.) et JANOT (C.), C. R. Acad. Sci., Paris, 1960, 250, 2007.

[52] Maystre (D.) et Petit (R.), Opt. Commun., 1972, 4, 380 .

[53] Maystre (D.) et Petit (R.), Principe d'un spectromètre à réseaux à transmission constante, Opt. Commun., à paraître.

NB. - Cette liste de références qui n'a pas la prétention d'être exhaustive devrait cependant constituer un guide suffisant pour le lecteur qui désirerait entreprendre une documentation systématique. On pourrait, dans ce cas, utiliser le compte rendu du Symposium de Tbilissi (sept. 1971, éditions Nauka, Moscou) comme point de départ d'une bibliographie qui s'intéresserait aux articles publiés en langue russe ou qui voudrait sortir du cadre des réseaux pour une étude plus générale de la diffraction des ondes électromagnétiques. 